\title{
Review Paper \\ Physical Fitness and Health-Related Physical Activity Programs in People With Down Syndrome: A Systematic Review
}

\author{
*Saeid Bahiraei ${ }^{1}$ (), Hassan Daneshmandi ${ }^{1}\left(\mathbb{C}\right.$, Rahman Amiri $^{1}$ (1)
}

1. Department of Sport Injuries and Corrective Exercises, Faculty of Physical Education, Guilan University, Rasht, Iran.

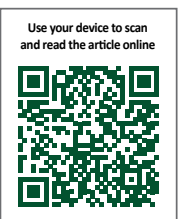

Citation: Bahiraei S, Daneshmandi H, Amiri R. [Physical Fitness and Health-Related Physical Activity Programs in People With Down Syndrome: A Systematic Review (Persian)]. Journal of Sport Biomechanics. 2020; 5(4):200-215. https://doi. org/10.32598/biomechanics.5.4.1

https://doi.org/10.32598/biomechanics.5.4.1

Key words:

Down syndrome,

Physical fitness, Physi-

cal activity

\section{ABSTRACT}

Objective Down Syndrome (DS) is the most common genetic disease in people with intellectual disability, with a prevalence of 1 in 800-1000 live births. Youth and adolescents with DS are a unique population in terms of health-related physical fitness factors.

Methods In this review study, to investigate the physical fitness factors and physical activity programs for youth and adolescents with DS, a search was conducted in international and national databases such as EBSCO, Medline, PubMed, Elsevier, Google Scholar and SID among studies conducted during 1996-2016 using following keywords in Persian and English: Physical fitness, physical activity, Down syndrome, rehabilitation, intellectual disability, muscular strength, muscular endurance, aerobic fitness, balance, agility and flexibility.

Results Initial search yielded 130 articles. After removing duplicates, 35 were remained for the review. The studies indicated lower cardiorespiratory capacity and physical fitness in people with DS than in their normal peers in strength, endurance, balance and agility as well as increased subcutaneous fat and body fat distribution.

Conclusion Adolescents and youth with DS have low cardiovascular and muscular capacity and physical fitness, overweight/obesity, and reduces health-related physical activities. Future studies should focus on strength testing and training protocols, methods for determining physical activity levels, and practical interventions to increase physical activities in DS patients.

\section{Extended Abstract}

\section{Introduction}

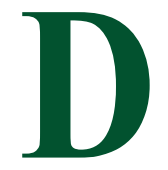

own Syndrome (DS) is the most common genetic disease in people with Intellectual Disability (ID), with a prevalence of 1 in 800-1200 live births [1-4]. Studies show that almost one-third of people with ID are physically active enough. Demographics and health profiles of these patients include congenital heart disease, respiratory diseases, deterioration of functional capacities due to Alzheimer's, recurrent pneumonia, sensory disorders, mus- culoskeletal disorders, and movement disorders. Physical fitness of people with ID is much lower than of the general population. People with DS are no exception. One of the important features of these patients is low physical activity [5].

Various studies have shown that DS patients have less physical activity than other people in the society and even other people with ID [6-9]. They, equal to or more than healthy people, need physical activity and the health of the cardiovascular and musculoskeletal systems to be healthy and prevent from diseases and premature death [10]. Low levels of physical fitness and function may be due to an increase in the prevalence of overweight or obesity as well

\section{* Corresponding Author:}

Saeid Bahiraei, PhD.

Address: Department of Sport Injuries and Corrective Exercises, Faculty of Physical Education, Guilan University, Rasht, Iran.

Tel: +98 (918) 7069265

E-mail: saeid_bahiraei86@yahoo.com 
as a decrease in bone mass growth which may eventually lead to the exacerbation of clinical manifestations. Therefore, the goal of all health organizations is to improve the quality of life and health of citizens. Given the effects of exercise and physical activity on increasing quality of life, social skills and reducing the risk of disease, it is necessary to study the effect of physical activity on indicators related to the health of young people and children with DS.

\section{Methods}

In this review study, to investigate the effect of physical activity on health of young people and children with DS, search was conducted in national and international databases including EBSCO, Medline, PubMed, Elsevier, Google Scholar and SID using following keywords in Persian and English: Physical Fitness, Physical Activity, Down Syndrome, Rehabilitation, Intellectual Disability, Muscle Strength, Muscle Endurance, Aerobic Fitness, Balance, Agility, and Flexibility. Initial search yielded 130 papers. Of these, 35 were selected based on inclusion and exclusion criteria.

\section{Results}

Table 1 presents a systematic review of studies that have shown physical fitness, cardiovascular fitness, strength and combined exercises, postural control, and body composition of individuals with DS. The results show that the cardiovascular fitness was lower among people with DS compared to people without ID. However, a selected exercise program could have a significant effect on improving their maximal oxygen uptake, peak minute ventilation, chest fluid content, cardiac output, mean arterial blood pressure, regular vascular resistance, regular vascular resistance index, and other cardiorespiratory parameters. Spending more time in moderate physical activity is associated with greater cardiorespiratory fitness in adolescents with DS.

The results showed an improvement in strength of lower extremity muscles and all muscle groups as well as cardiac function and agility compared to the controls. People with DS have unstable postural control and increased oscillation in both anterior-posterior and medio-lateral directions compared to normal people. In anterior-lateral plane with eyes open and closed conditions, DS people with and without visual information had more body sway than healthy individuals in the lateral and anterior-posterior plane. The prevalence of overweight is higher in these patients than in the general population; children with DS are often overweight and obese compared to the general population.

\section{Conclusion}

One of the reasons for the lower cardiovascular fitness in DS people can be due to less muscle mass, less muscle strength, thyroid disorders, muscle laxity, obesity or sym-

Table 1. Systematic review of some studies related to the physical fitness factors in people with DS

\begin{tabular}{|c|c|c|c|}
\hline Author (s) & Method & Findings & Conclusions \\
\hline $\begin{array}{l}\text { González-Agüero et } \\
\text { al. (2014) }\end{array}$ & $\begin{array}{l}21 \text { weeks of circuit training, } \\
\text { including plyometric jumps }\end{array}$ & $\begin{array}{c}\text { Exercise group increased all their cardiore- } \\
\text { spiratory parameters compared to baseline } \\
\text { after } 21 \text { weeks of training. This group } \\
\text { showed higher values than the control } \\
\text { group in all cardiorespiratory parameters } \\
\text { after training }\end{array}$ & $\begin{array}{l}\text { Youths with DS achieved improve- } \\
\text { ments in several cardiorespira- } \\
\text { tory parameters after } 21 \text { weeks } \\
\text { of training including plyometric } \\
\text { exercises }\end{array}$ \\
\hline $\begin{array}{l}\text { Shields and Taylor } \\
\text { (2010) }\end{array}$ & $\begin{array}{l}\text { Performing } 6 \text { exercises using } \\
\text { weight machines performed } \\
\text { twice a week for } 10 \text { weeks ( } 3 \\
\text { sets of } 12 \text { reps) }\end{array}$ & $\begin{array}{l}\text { Improvement in lower limb muscle strength } \\
\text { was observed compared to the control } \\
\text { group. No major adverse events were } \\
\text { recorded }\end{array}$ & $\begin{array}{c}\text { Progressive resistance training is a } \\
\text { feasible and safe exercise option } \\
\text { that can improve lower limb } \\
\text { muscle strength in adolescents } \\
\text { with DS }\end{array}$ \\
\hline Rigoldi et al. (2011) & $\begin{array}{l}\text { An analysis of the centre of } \\
\text { pressure (COP) displacement } \\
\text { in both time and frequency } \\
\text { domains during standing posi- } \\
\text { tion was performed for the } \\
\text { three groups of subjects }\end{array}$ & $\begin{array}{l}\text { COP medio-lateral range of motion pointed } \\
\text { out a decrease for both groups (pathological } \\
\text { and control) in time domain analysis, but DS } \\
\text { people showed a larger frequency of move- } \\
\text { ment in medio-lateral direction in frequency } \\
\text { domain analysis }\end{array}$ & $\begin{array}{l}\text { DS people focused on overcom- } \\
\text { ing the lack of balance caused by } \\
\text { hypotonia and ligament laxity, } \\
\text { while control group attempted to } \\
\text { improve their strategy using differ- } \\
\text { ent strategy development. }\end{array}$ \\
\hline $\begin{array}{l}\text { Frey and Chow } \\
\qquad(2006)\end{array}$ & $\begin{array}{l}\text { Physical fitness was assessed } \\
\text { using 6- (ages 6-8 years) or } \\
\text { 9- (ages 9-18 years) minute } \\
\text { run, sit-up, isometric push-up, } \\
\text { sit and reach, and sum of } \\
\text { skinfold }\end{array}$ & $\begin{array}{l}20 \% \text { of samples were classified as over- } \\
\text { weight/obese. Overweight/obesity is mini- } \\
\text { mally associated with aerobic fitness and } \\
\text { muscular strength, but BMI had no impact } \\
\text { on endurance and flexibility or motor skills }\end{array}$ & $\begin{array}{l}\text { Overweight/obesity reduce aero- } \\
\text { bic fitness and muscular strength }\end{array}$ \\
\hline
\end{tabular}


pathetic system disorders in response to exercise, inactive lifestyle, eating habits, lack of recreational activities, lack of motor coordination, lack of sufficient motivation for physical activity. Some studies have shown that proper exercise can improve cardiovascular health. One of the most important types of exercises used for people with DS is strength training. The results have shown that the implementation of progressive strength training improves the strength of the muscles in the body [15-18], increases muscle mass and decreases weight [17], improves functional tasks and maximal aerobic capacity [15] in individuals with DS [19].

Decreased muscle tone and joint laxity are common in people with DS. In DS patients, due to some factors such as the number of muscle fibers or a lower percentage of slowtwitch muscle fibers, muscle strength is poorer than in normal and mentally retarded people. This muscle weakness in the lower limbs and poor postural balance increase the risk of falling in these people $[1,4]$. Body mass index and body fat percentage are also higher in people with DS than in healthy people. Overweight and obesity are new concerns for DS patients. Some studies have shown that $35-50 \%$ of them are obese. The main reason for their overweight is a sedentary lifestyle, of course because these people are not able to participate in health promotion activities and programs due to many obstacles.

\section{Ethical Considerations}

Compliance with ethical guidelines

This article is a meta-analysis with no human or animal sample.

Funding

This research did not receive any grant from funding agencies in the public, commercial, or non-profit sectors.

Authors' contributions

Conceptualization, methodology and supervision: all authors; Review and writing the original draft and sources: Saeed Bahiraei, Rahman Amiri; Review and editing: Hassan Daneshmandi.

\section{Conflicts of interest}

The authors declared no conflict of interest.

\section{Acknowledgements}

The authors would like to thank the research and educational officials of the University of Guilan and the Faculty of Physical Education and Sports Sciences. 


\title{
بررسى و مطالعه برنامههاى أمادتى جسمانى و فعاليت بدنى مرتبط با سلامت در افراد سندرم داون: مرورى نظاممند بر مطالعات تخذشته
}

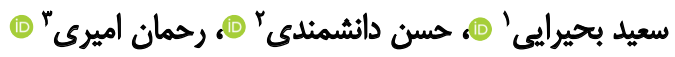 \\ 1. كروه آسيب شناسى ورزشى وحركات اصلاحى، دانشكده تربيت بدنى و علوم ورزشى، دانشعاه كيلان، رشت، ايران.
}

\begin{abstract}
حكيد

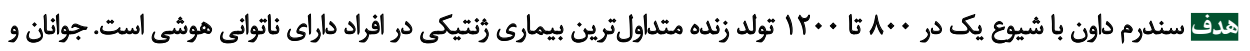

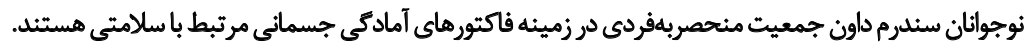

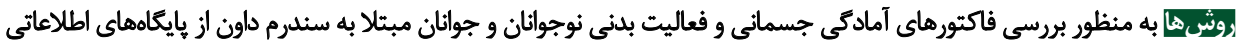

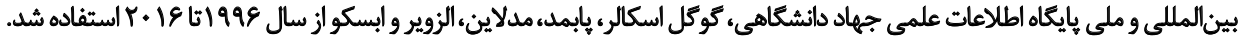

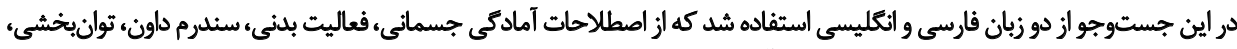

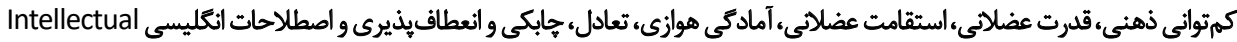
Disability, Down syndrome, Physical fitness, Rehabilitation, Muscles Strength and Endurance, Balance Physical activity,flexibility,aerobic capacity

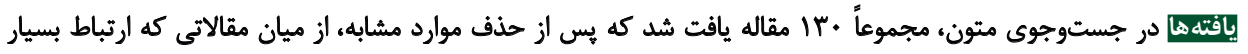

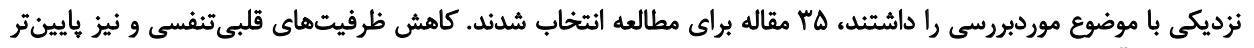

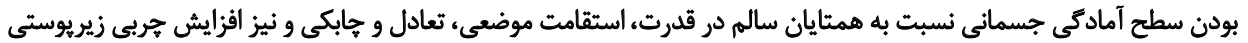

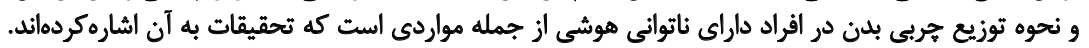

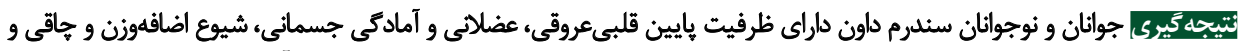

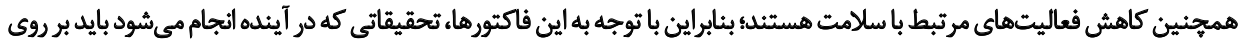

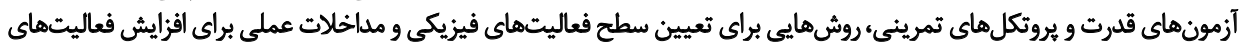

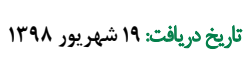

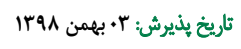

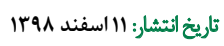

دانستهاند كه هN درصد اين افراد، ناتوانان هوشي خفيف هستيد

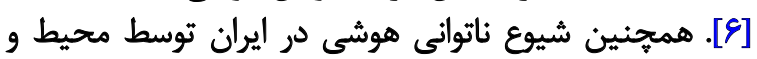

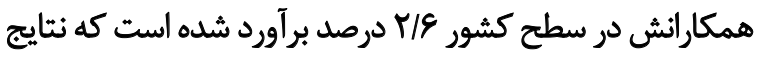

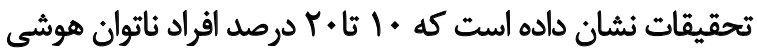

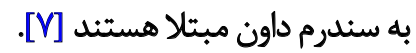

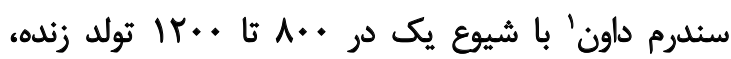

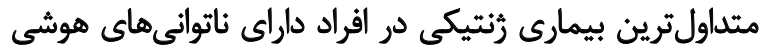

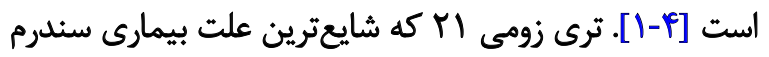

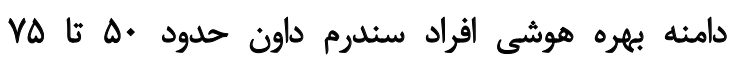

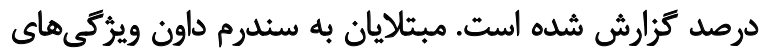

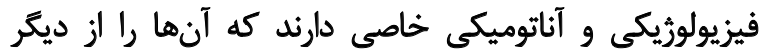

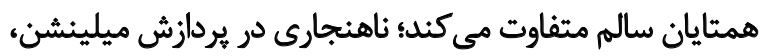

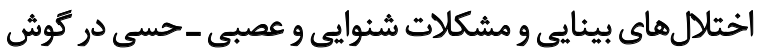
داخلى، از اختلالهاي افراد مبتلا به سندرم داون است. بيش

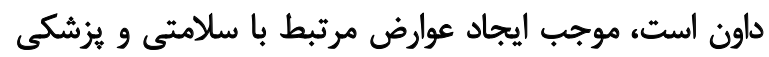

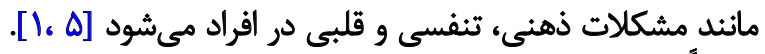

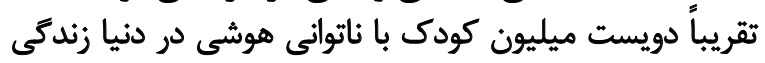

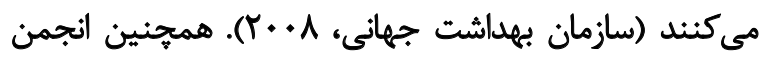

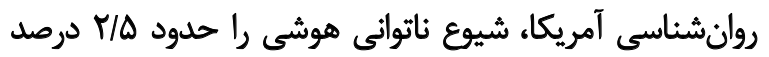

1. Down syndrome

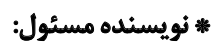
سعيد بحيرايي نشانى: رشت، دانشكاه كيلان، دانشكده تربيت بلدنى و علوم ورزشي، كروه آسيب شئاسى ورزشى و حركات اصلاحى. تلفن:

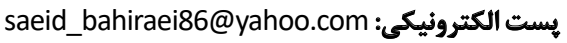




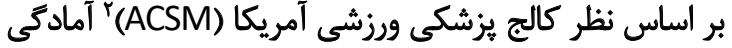

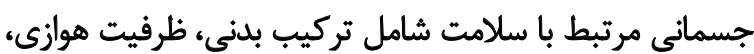

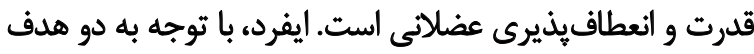

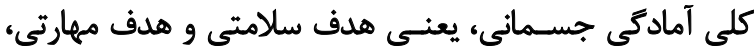

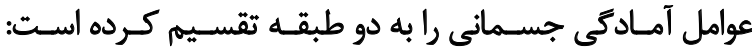

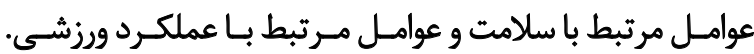

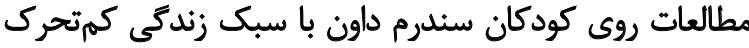

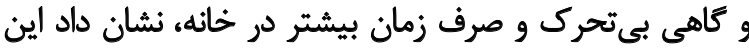

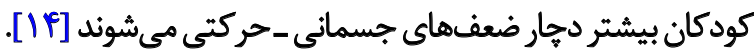

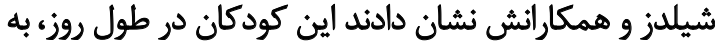

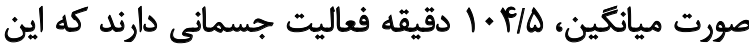

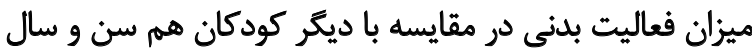

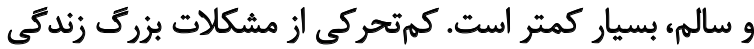

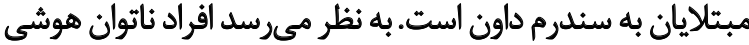

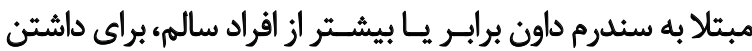

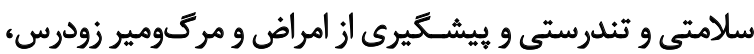

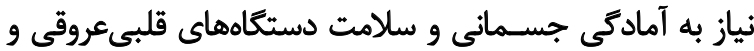

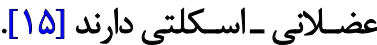

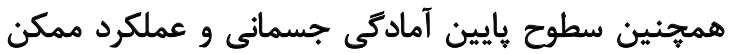

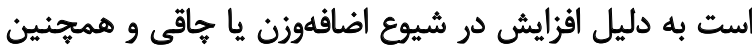

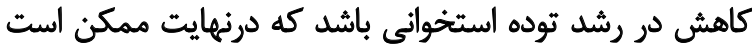

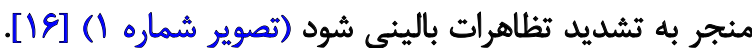

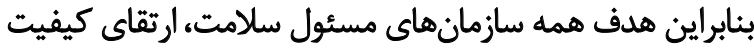

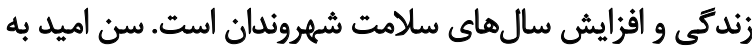

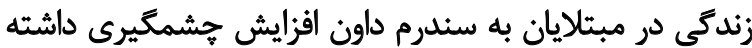

2. The American College of Sports Medicine
از هشتاد ويرَّى بالينى در افراد با سندرم داون شرح داده شده

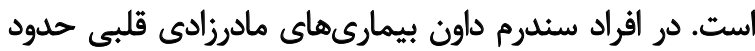

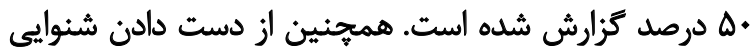

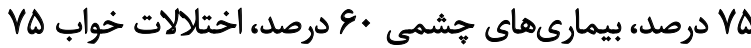

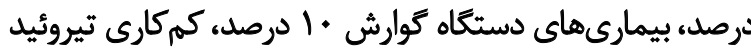

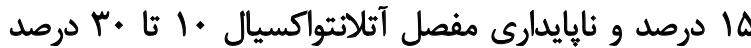

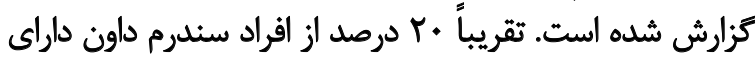

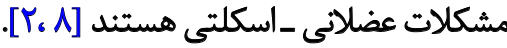

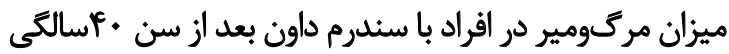

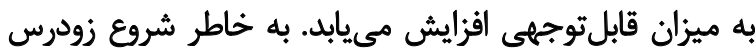

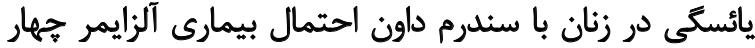

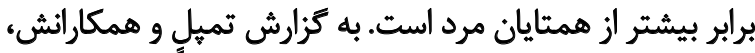

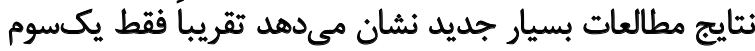

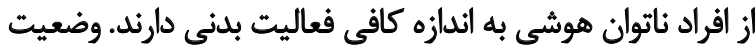

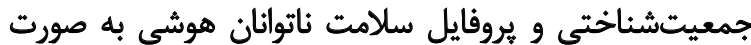

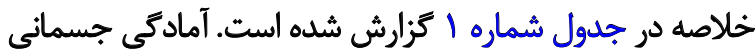

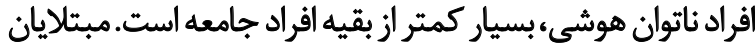

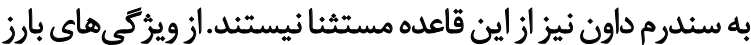

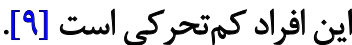

نتايج تحقيقات مختلف نشان داده است اين افراد نسبت به به

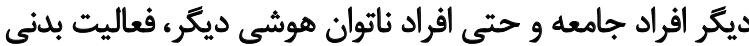

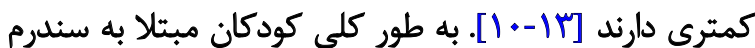

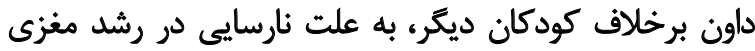

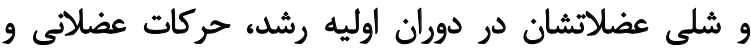

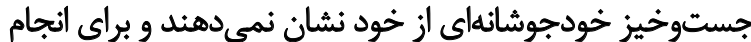

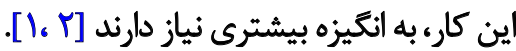

جدول ا. توضيحات جمعيتشثاختي و بروفايلهاي سلامت در افراد مبتّلا به سندرم داون

\begin{tabular}{|c|c|}
\hline ويؤكى هائ1) & توضيحات \\
\hline 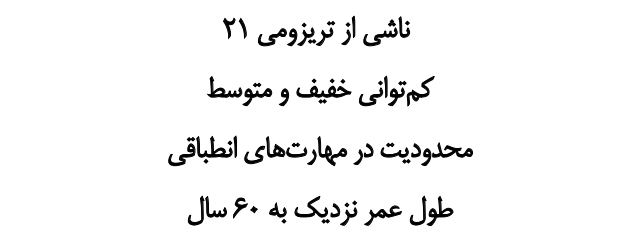 & توضيحات جمعيتشناختى \\
\hline 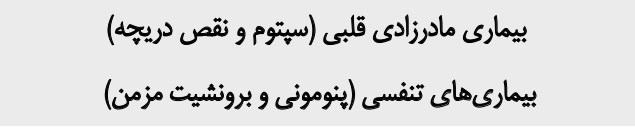 & يروفايل سلامت كودكان \\
\hline 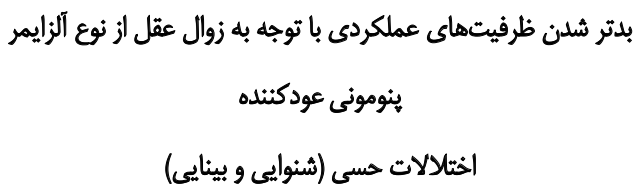 & بزركسالان \\
\hline اختلالات عضلانى ـ اسكلتى(نايايدارى مفاصل و يوكى استخوان) & \\
\hline
\end{tabular}

مجله بيومكانيك ورنث 


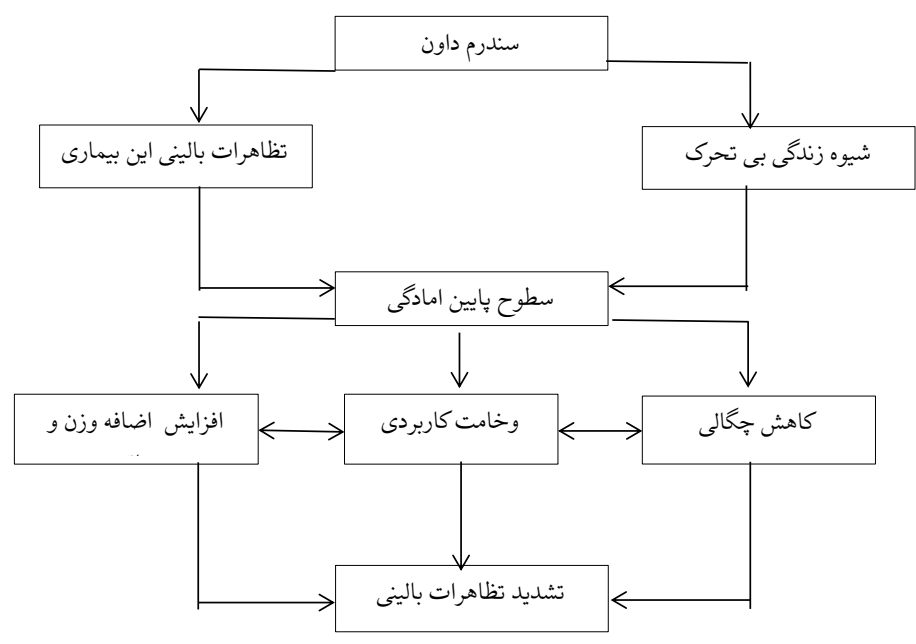

مجله بيومكانيك وزنش

علمى جهاد دانشعاهي، كوكل اسكالر، يابمد، مدلاينء الزوير و

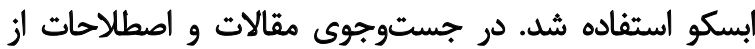

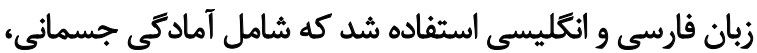

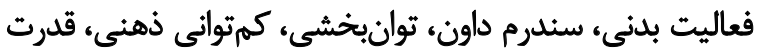

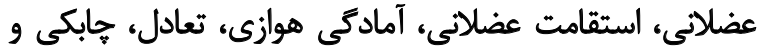

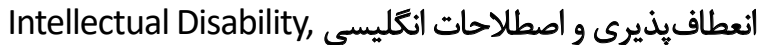
Down syndrome, Physical fitness, Rehabilitation, Muscles strength and endurance, Balance, Physical activity, flexibility, aerobic

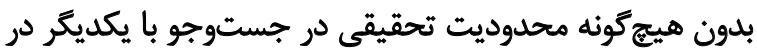

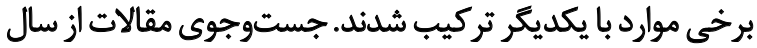

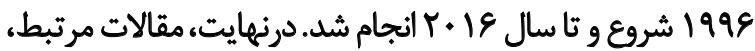
براي بررسى سيستماتيك مورد تجزيه و تحليل قرار كرفتند. در

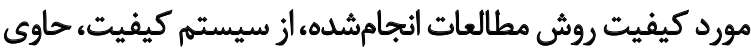

تصوير ا.ارتباط آمادكى جسمانى و تظاهرات بالينى در افراد سندرم داون

است؛ بنابراين، تغيير در عملكرد و ساختار بدني در مبتلايان به

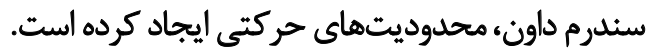

اين مشكلات آثار منفى در كيفيت زندكى، هم در خصوص

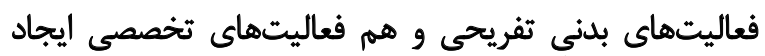

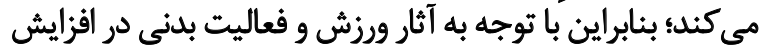

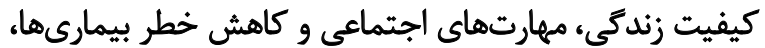

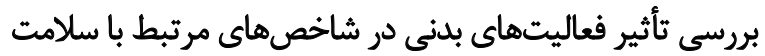

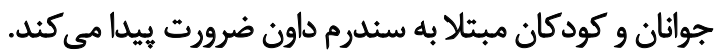

\section{مواد ورش مشا}

در روند اين تحقيق به منظور بررسى فاكتورهاى آمادكى

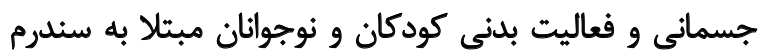

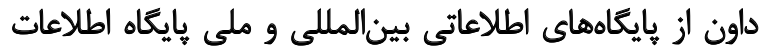

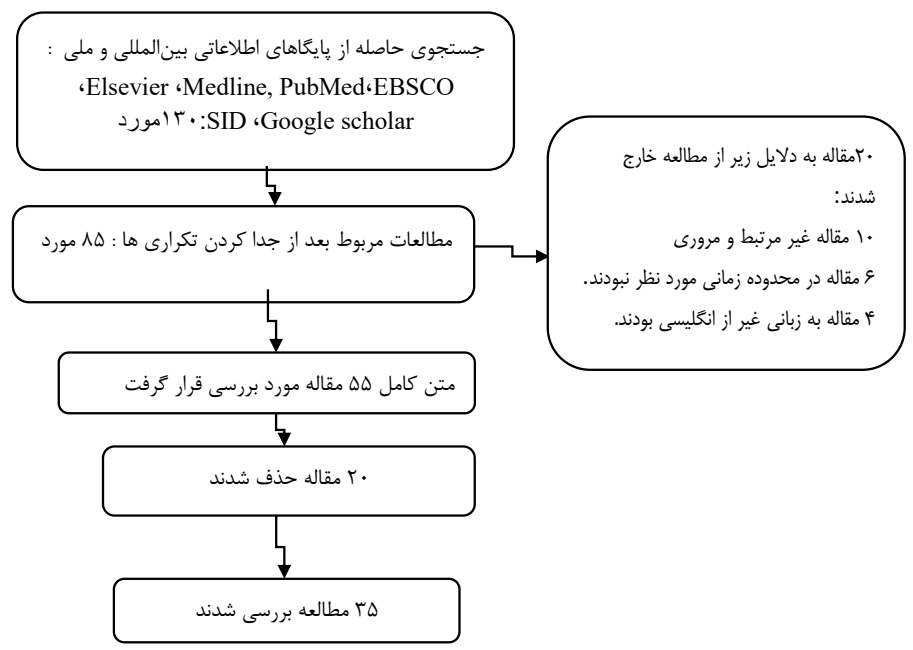

مجله بيومكانيك ورنشي

تصوير ז. مراحل جستوجوى متون و بازيابى مقالات 
جدول r. بازبينى نظاممند آمادتى جسمانى قلبىعروقى در افراد سندرم داون

\begin{tabular}{|c|c|c|c|c|c|}
\hline تفسير & خلاصهنتايج & روثُ اندازهيّيرى & آزمودنى تهداد & عنوان تحقيق & محققلن \\
\hline 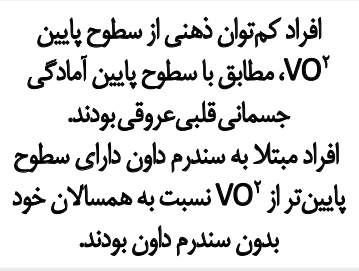 & 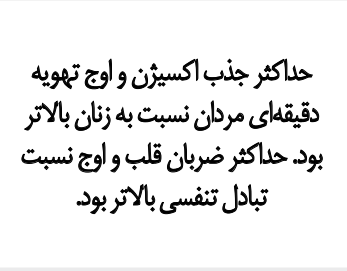 & بروتكل راه رقتن بر روى تردميل & إزمودنى & 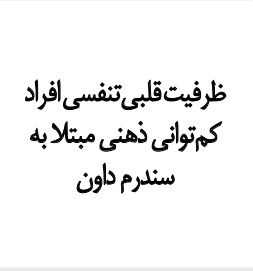 & $=\frac{\frac{c}{8}}{\sum}$ \\
\hline 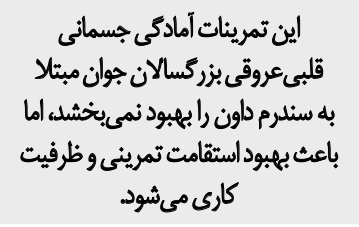 & 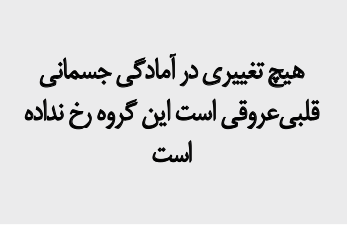 & 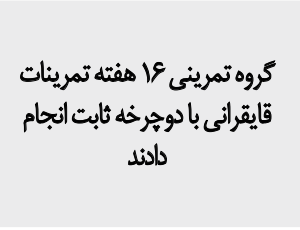 & عاع هرد & 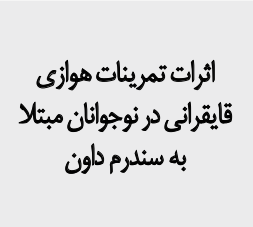 & 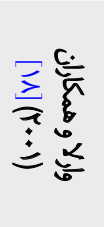 \\
\hline 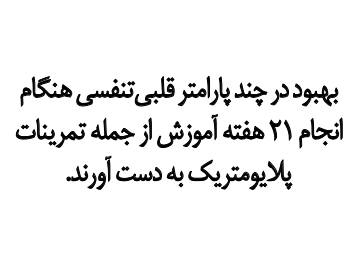 & 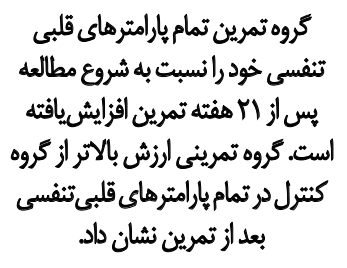 & 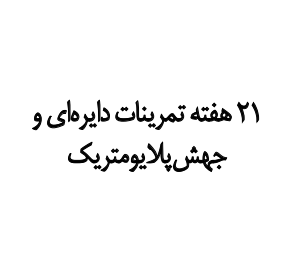 & 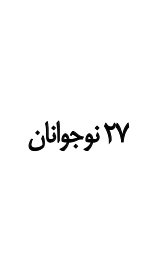 & 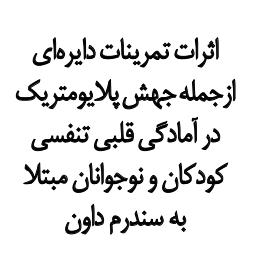 & 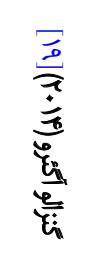 \\
\hline 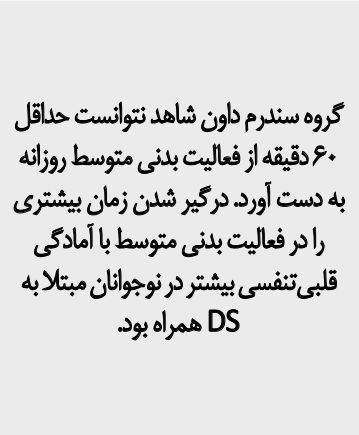 & 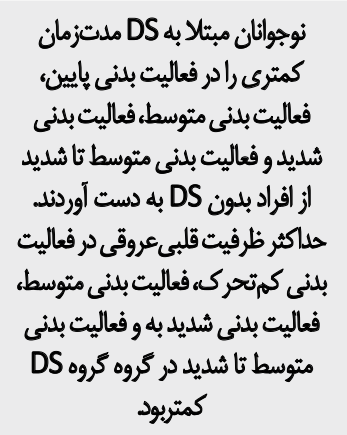 & 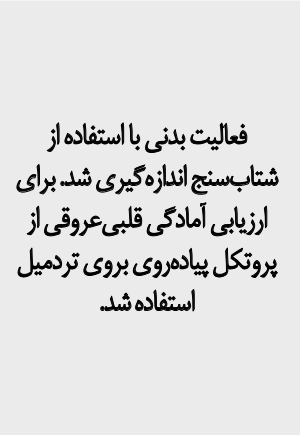 & كأنوجوانان & 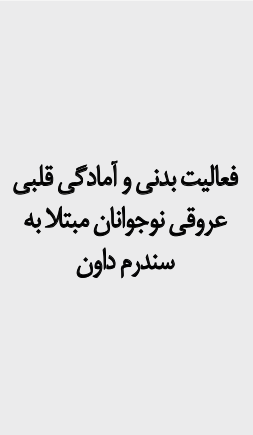 & 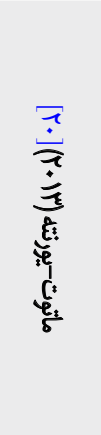 \\
\hline 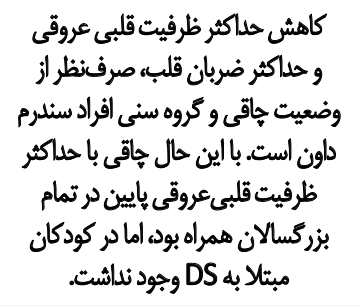 & 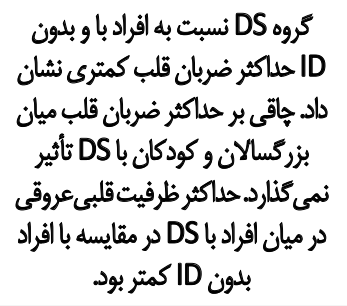 & أزمون تردميل و الز شاخص توده & مالون & 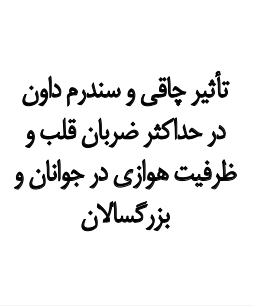 & 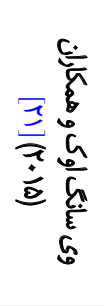 \\
\hline 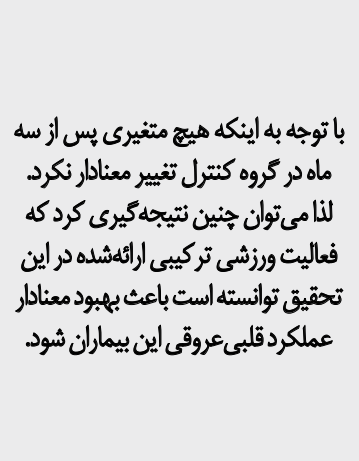 & 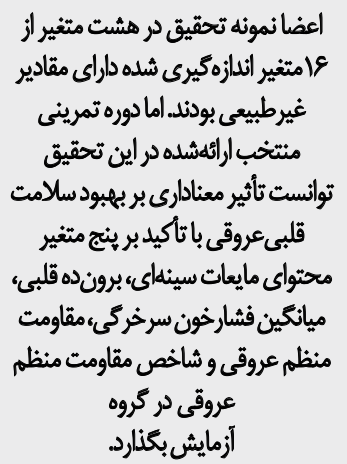 & 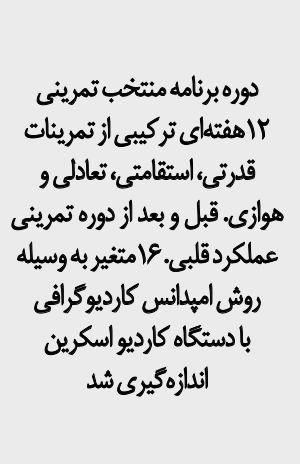 & 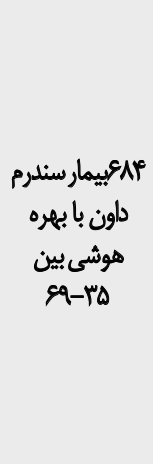 & 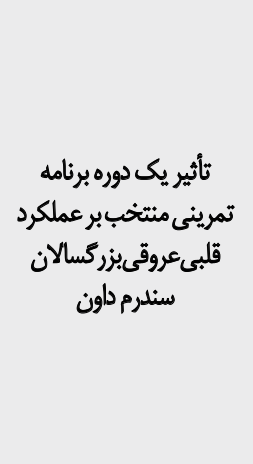 & 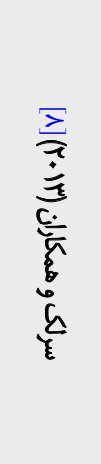 \\
\hline
\end{tabular}

مجله بيومكانيك ولنش 
جانبى در دو وضعيت با جشمان باز و بسته، افراد سندرم داون با بان بانيا

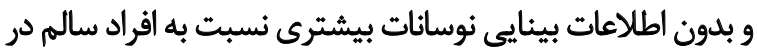
صفحه جانبى و قدامى خلفى داشتند.

جدول شماره ه بازبينى نظاممندى از تركيب بدنى در افراد

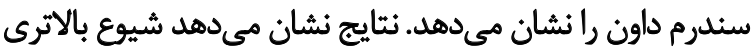

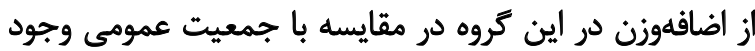

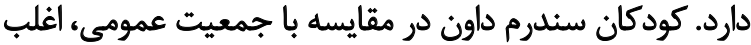

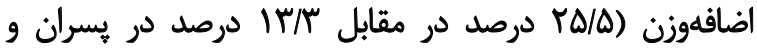

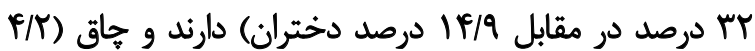

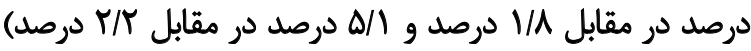

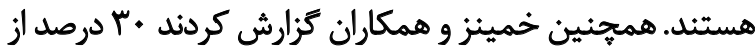

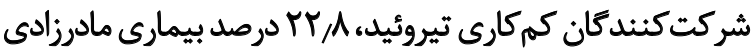

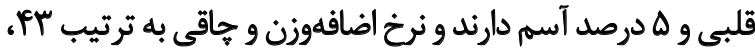

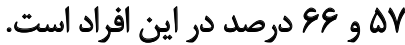

H

\section{أماديّى جسمانى قلبى عروقى}

يكى از دلايل كمتر بودن ظرفيت قلبىعروقى در اين افراد

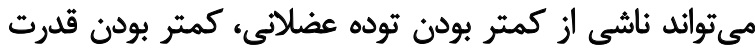

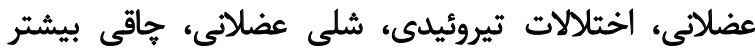

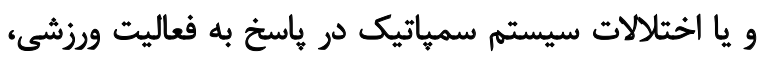

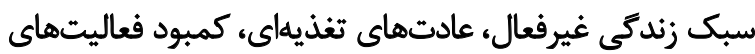

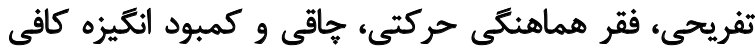

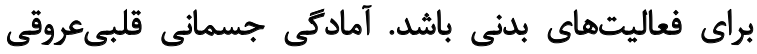

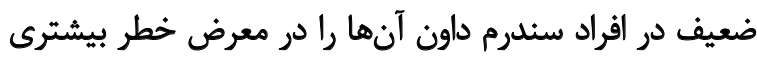

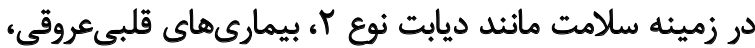

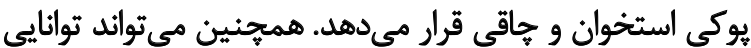

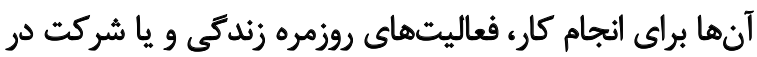

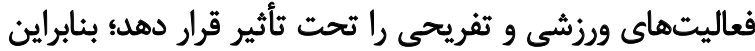

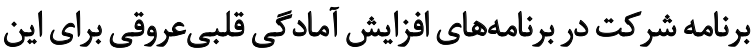

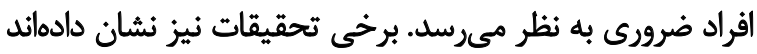

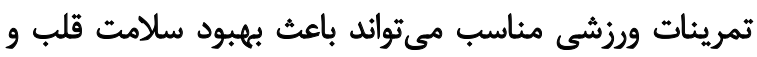

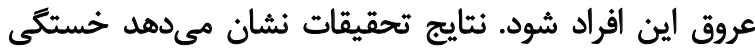

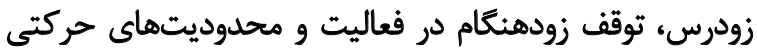
دليل عدم اثربخشى تمرينات ورزشيى در اين افراد است فيت.

تنايج جندين تحقيق انجامشده در اين زمينه نشان دادهاند

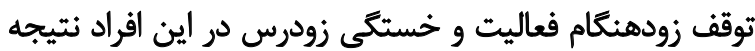

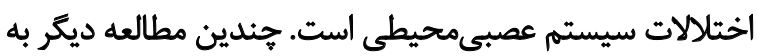

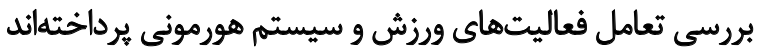

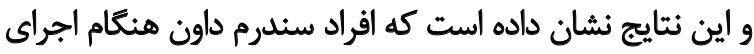

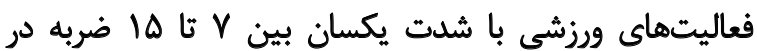
دقيقه ضربان قلب كمترى دارند. دليل اين موضوع مئن لتواند
جهار معيار جمعيت موردمطالعه (سندرم داون و ناتوانان هوشى)،

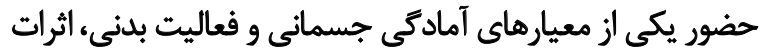

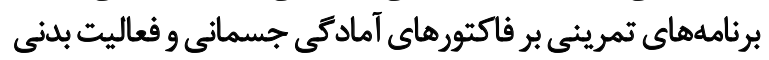

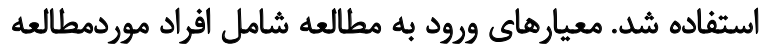

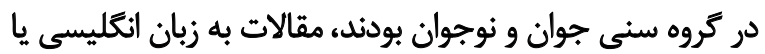

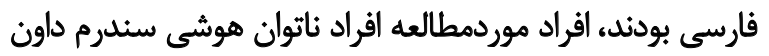

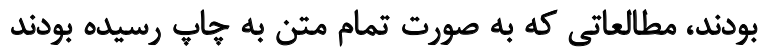

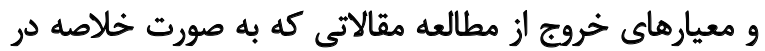

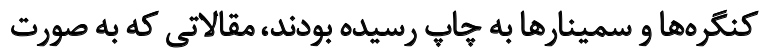

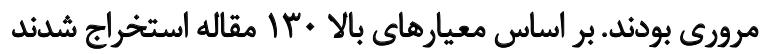

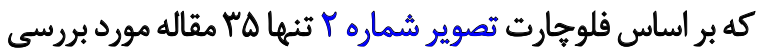
قرار كرفت (تصوير شماره ؟).

نتايج

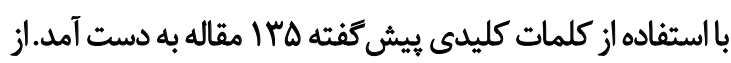

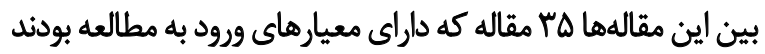

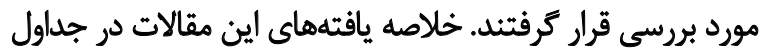

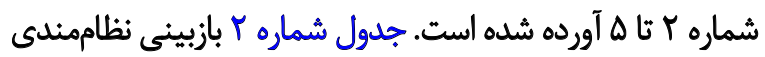
از آمادكى جسمانى قلبى عروقى افراد سندرم داون را نشان مى دهد.

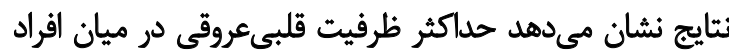

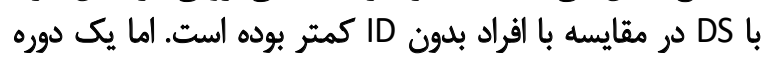

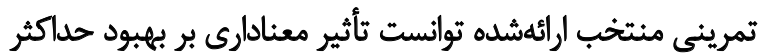

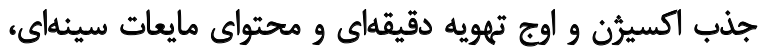

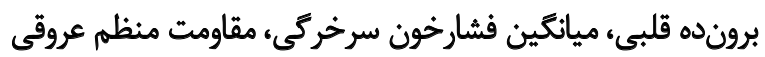

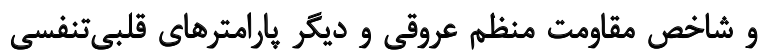

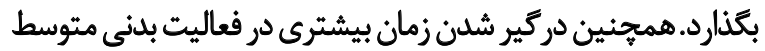

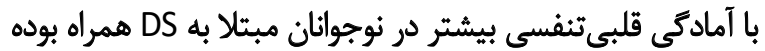

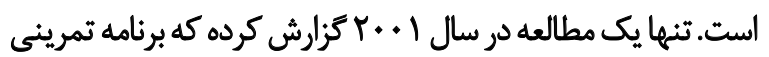

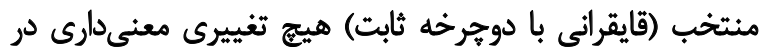

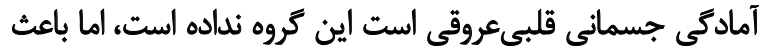
بهبود استقامت تمرينى وظرفيت قائي كارى اين افراد شده است.

جدول شماره با، بازبينى نظاممندى از تمرينات قدرتى و

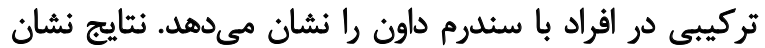

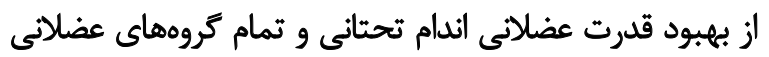

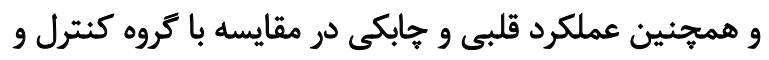

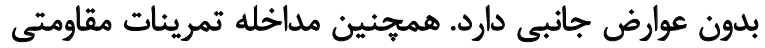

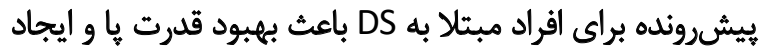
توانايى بالا رفتن از يله شده است.

جدول شماره f بازبينى نظاممندى از كنترل ياسجر (تعادل

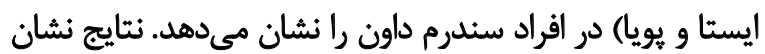

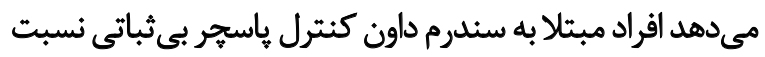

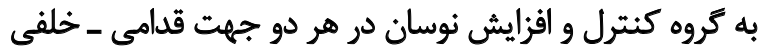

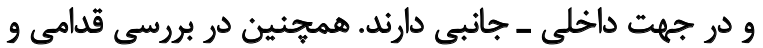


جدول ץ. بازبينى نظاممند تمرينات قدرتى و تركيبى در افراد سندرم داون

\begin{tabular}{|c|c|c|c|c|c|}
\hline تفسير & خلاصه نتايج & روش اندازهيرى & آزمودني تعدادا & عنوان تحقيث & محققان \\
\hline با يكى برنامه تركيبى هوازيى و قدرتى & 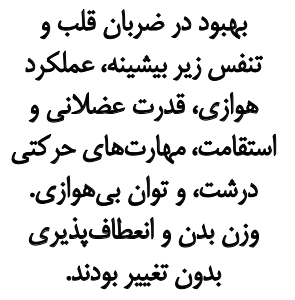 & 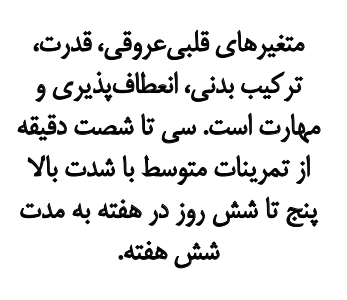 & 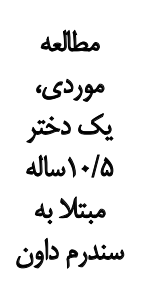 & 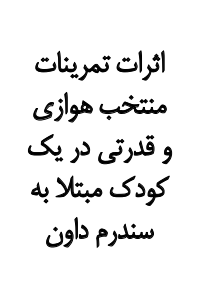 & 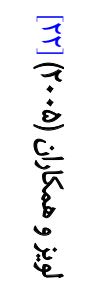 \\
\hline 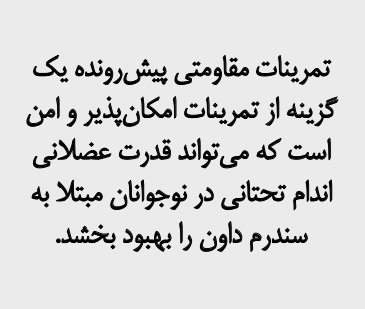 & 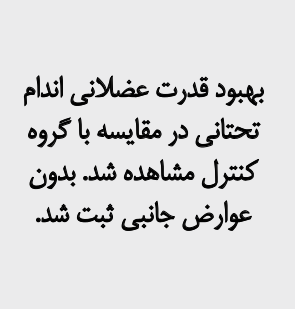 & 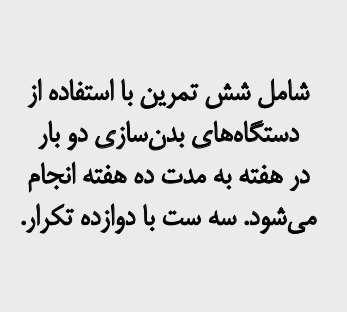 & 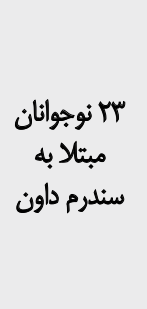 & 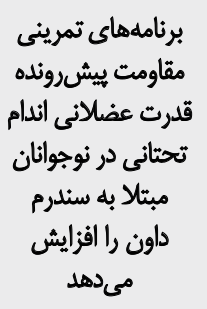 & 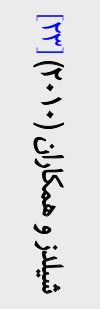 \\
\hline 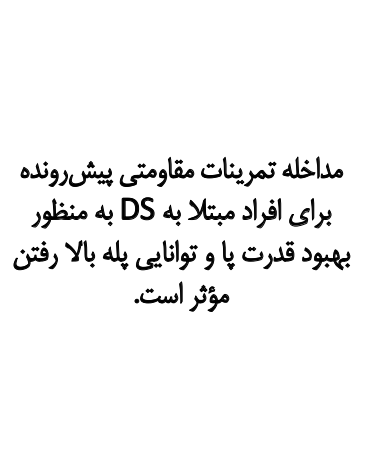 & 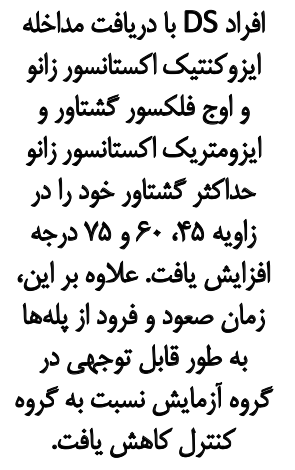 & 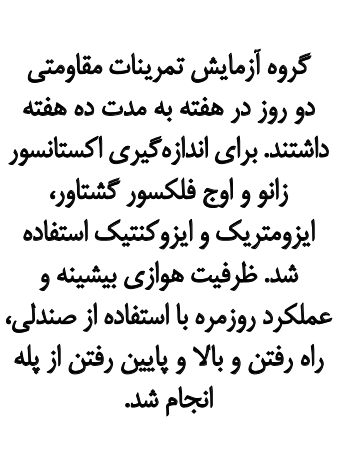 & سي نفر مبثلا & 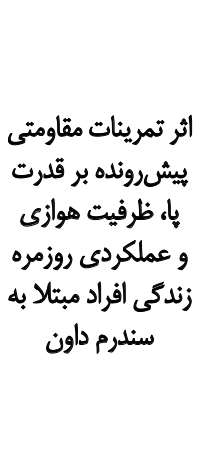 & 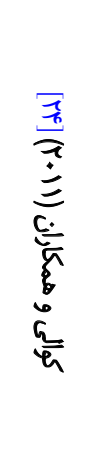 \\
\hline 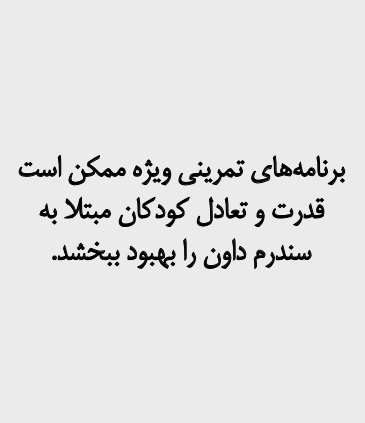 & 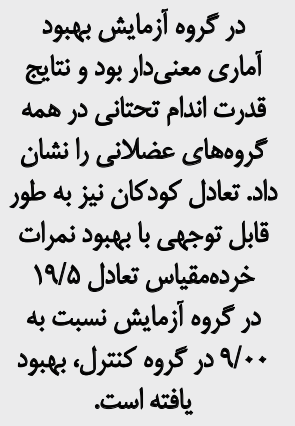 & 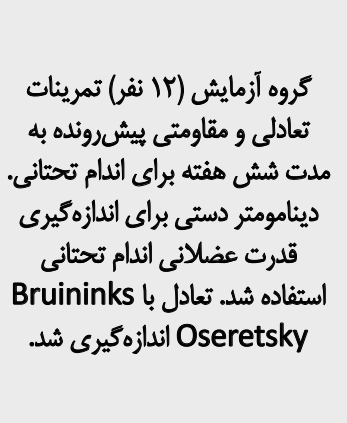 & 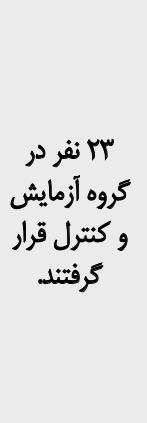 & 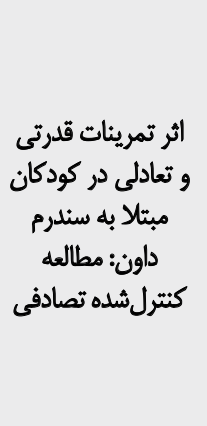 & 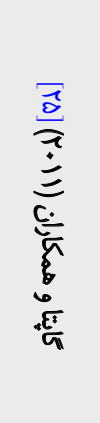 \\
\hline 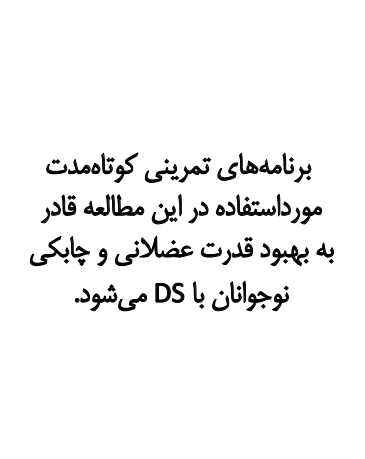 & 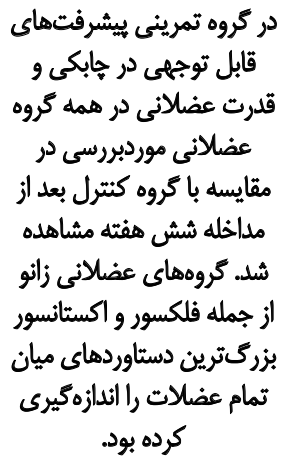 & 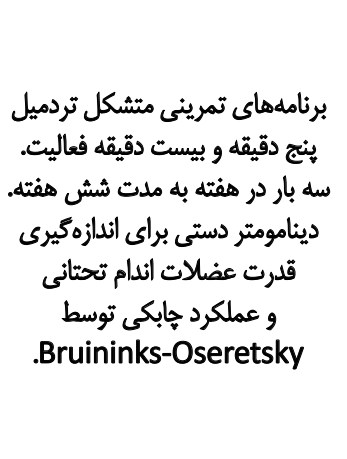 & 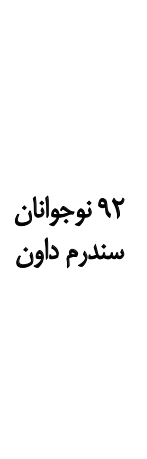 & 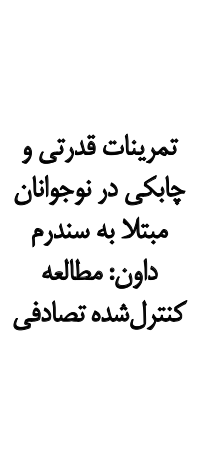 & 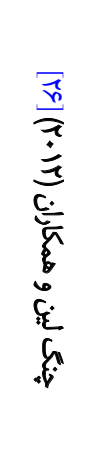 \\
\hline
\end{tabular}

مجله بيومكانيك وزنش 
جدول F. بازبينى نظاممند كتترل ياسجر در اقراد سندرم داون

\begin{tabular}{|c|c|c|c|c|c|}
\hline تفسير & خالا & روش & & عز & \\
\hline 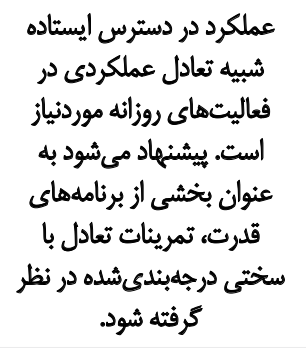 & 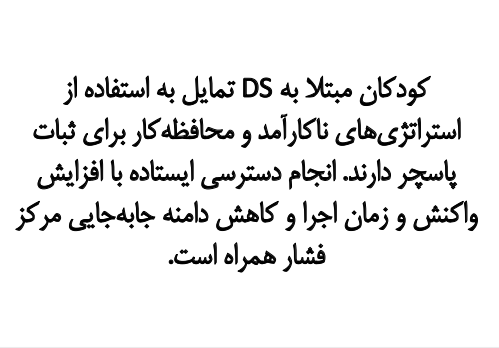 & 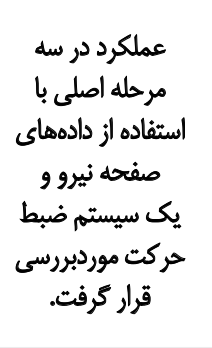 & 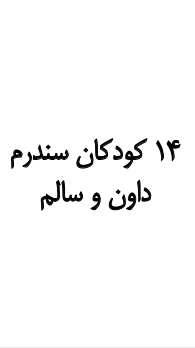 & 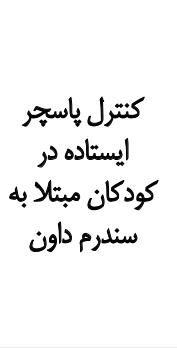 & 疍高 \\
\hline 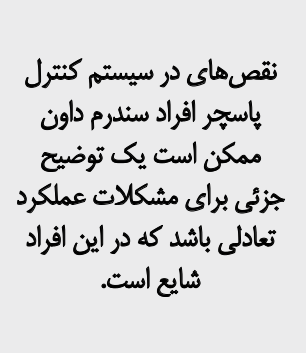 & 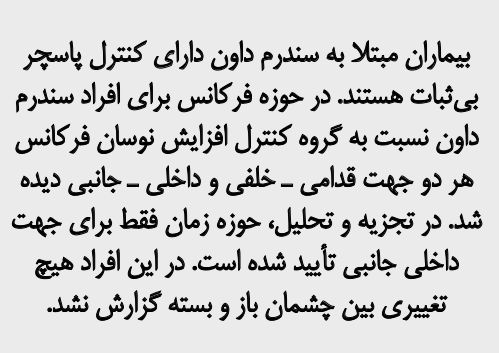 & 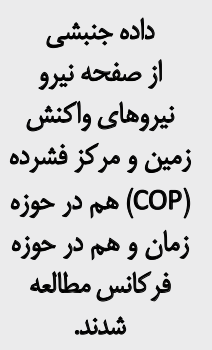 & 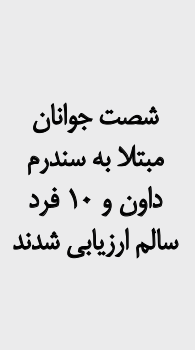 & 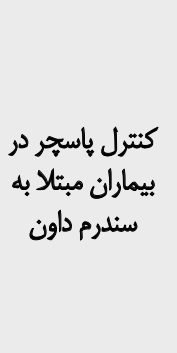 & 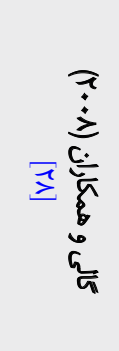 \\
\hline 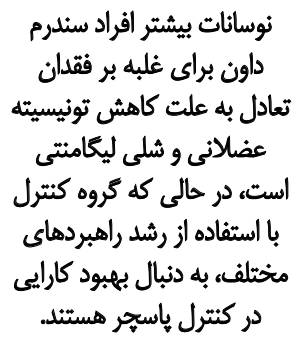 & 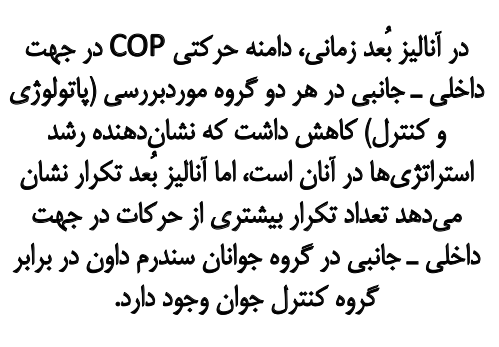 & 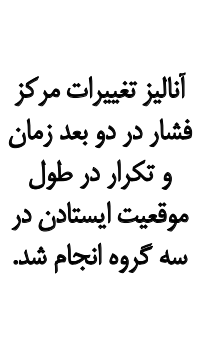 & آناليز كنترل ياسهر كره & 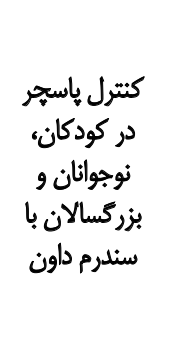 & 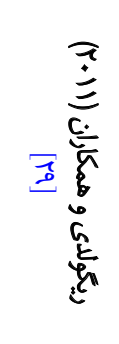 \\
\hline- & 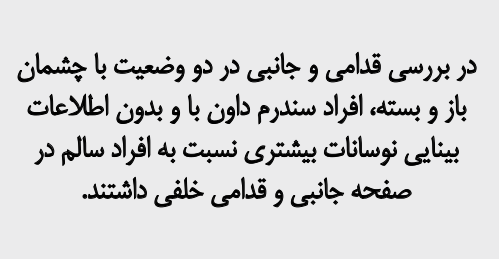 & 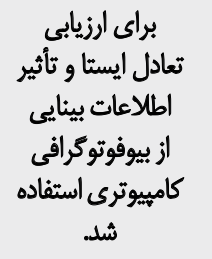 & دازئ ونه فرد سندرم & 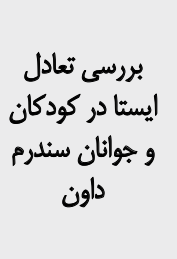 & 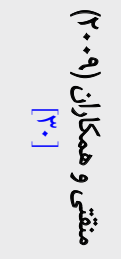 \\
\hline 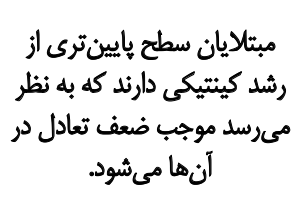 & 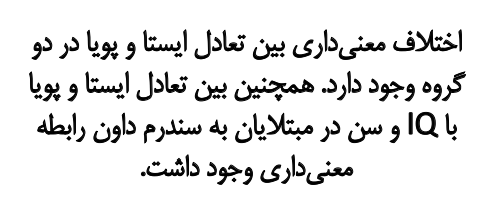 & 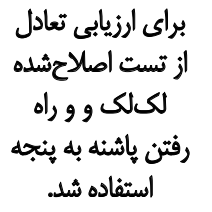 & دخي دانش آموز & 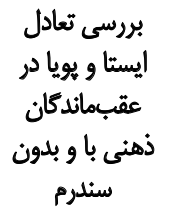 & 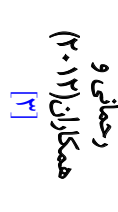 \\
\hline
\end{tabular}

مجله بيومكانيك ورنش

جدول ه. بازبينى نظاممند وضعيت تركيب بلئى در افراد سندرم داون

محققان

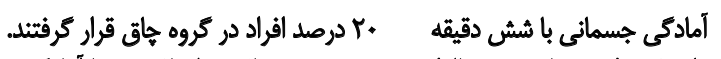

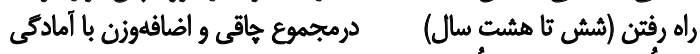

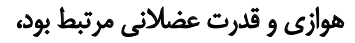

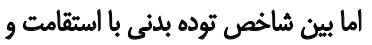

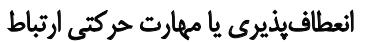
معنادار نبوده است.

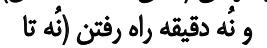

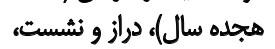

شناي ايزومتريك و ضُخامت درن

زيرجلدى الزيابي شلد.

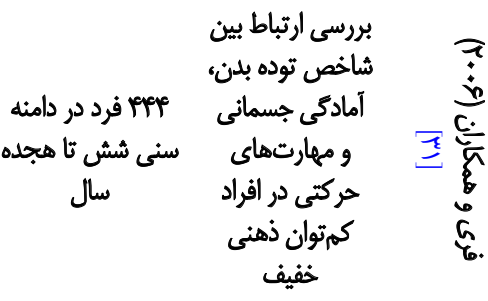




\begin{tabular}{|c|c|c|c|c|c|}
\hline تفسير & 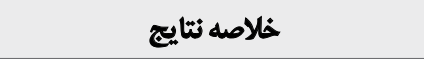 & روش & تعداد آزهودئي ها & عنوان تحقيث & محققان \\
\hline 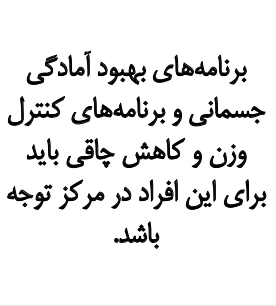 & 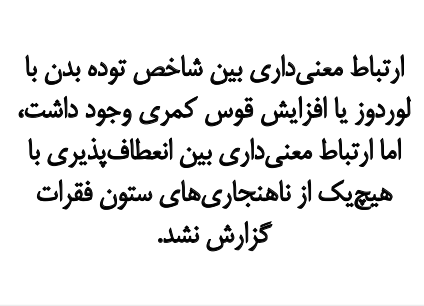 & 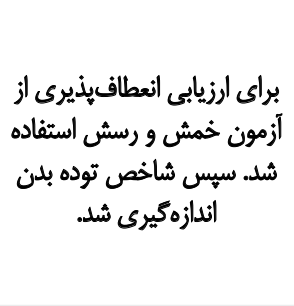 & دختر سنداه دانش آهوز & 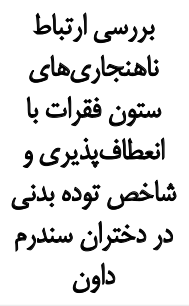 & 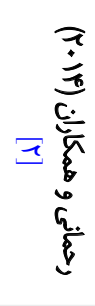 \\
\hline 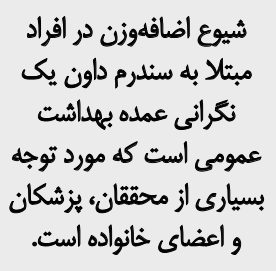 & شقيوع بالاترى از اضافهوزن در اين كروه در عمديت & شاخص تّده بلنى & 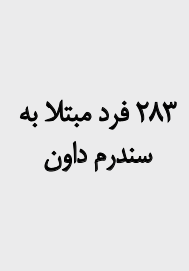 & شر افراد اضندافهوزن & 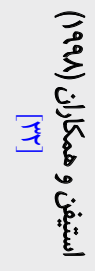 \\
\hline 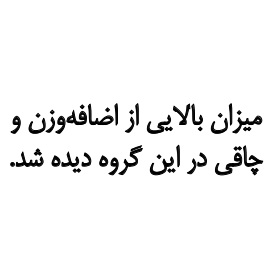 & 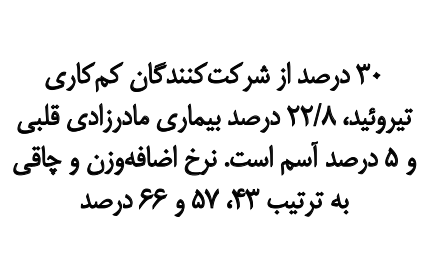 & شاخص توده بلدى & 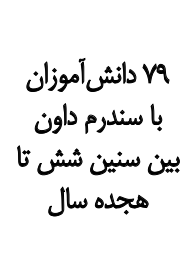 & 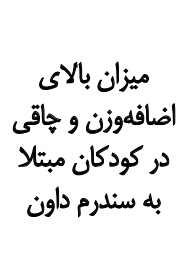 & 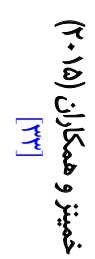 \\
\hline 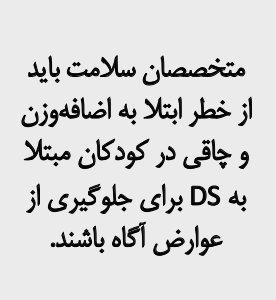 & 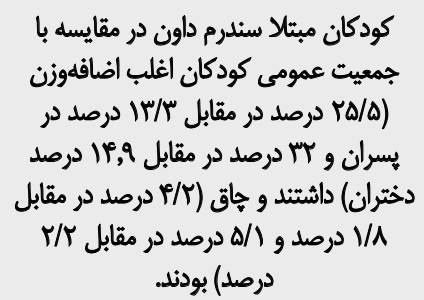 & 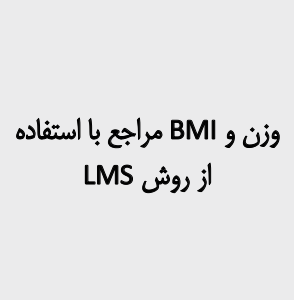 & عها نفر & 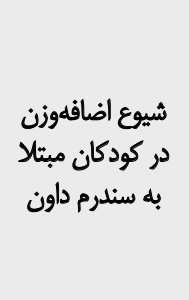 & 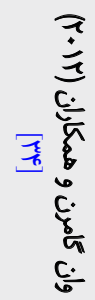 \\
\hline
\end{tabular}

مجله بيومكانيك وزنث

ها ممكن است يك عامل مهمم در حداكثر اكسيرن مصرفى در

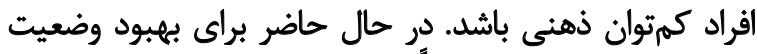

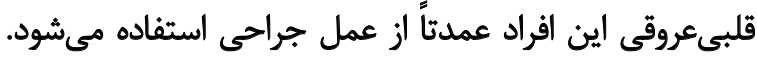

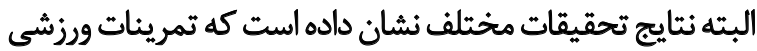

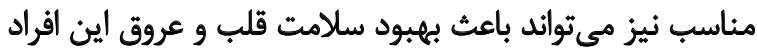

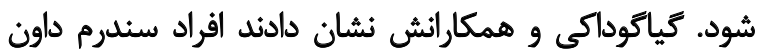

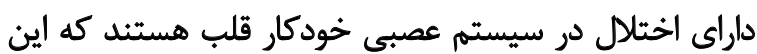

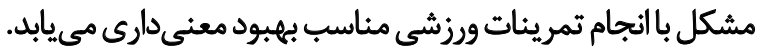

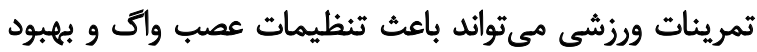

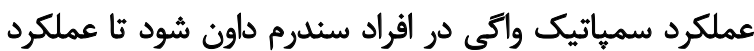

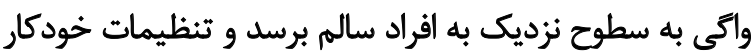
قلبى در اين افراد بهبود يابد [عَّ].

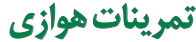

آندريولو و ديكران يس از مرور كليهتحقيقات انجامشده در

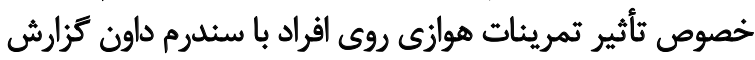
كردند كه مدارك كافى وجود ندارد كه تمرينات هوازئ بازي بتواند
عدم كاتكولامينها (ايى نفرين و نورايى نفرين) در فعاليتهاى

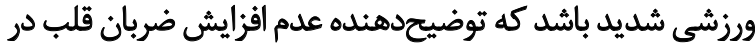

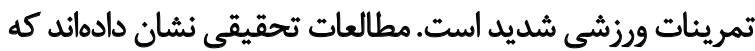

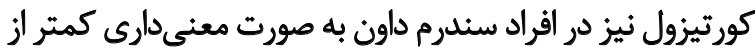

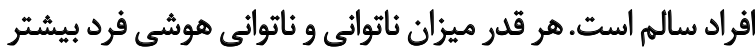

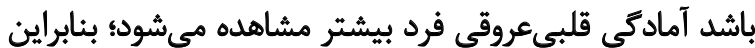

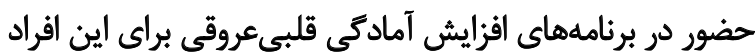
مى متواند بسيار مهمر و مؤثر باشد. تأثير تمرينات ورزشى بر بهبود ضربان قلب نتايج هاستور و همكارانش نشان داد ظرفيت تمرين، حداكثر إئر

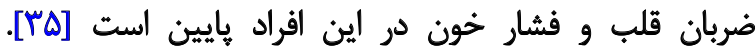

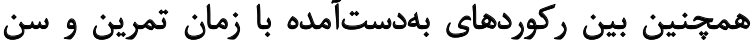

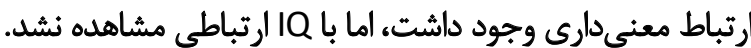

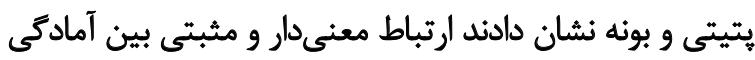

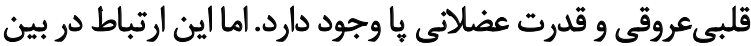
افراد سندرم داون بيشتر بود. نتايج اين مطالعه نشان داد قدان قدرت 
با تمرينات هوازى تركيب شود باعث نتايج مثبتى در سيستم

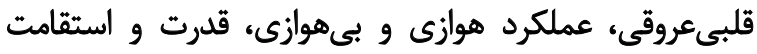

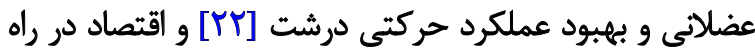

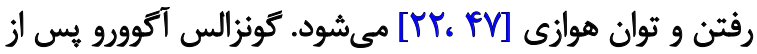

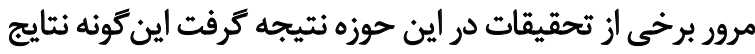

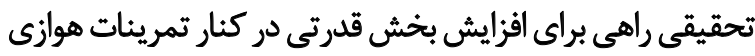

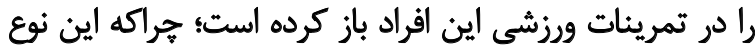

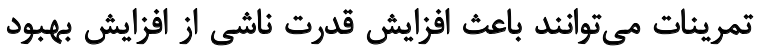

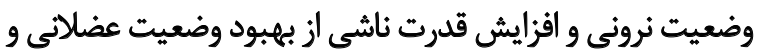

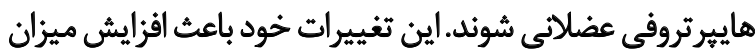

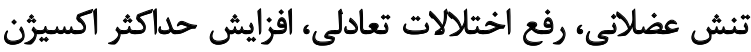

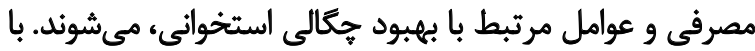

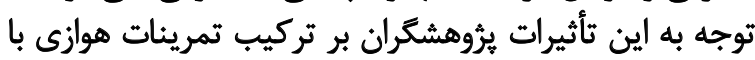

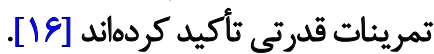

يكى از فوايد تمرينات قدرتى براى افراد سندرم داون آماده

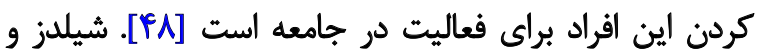

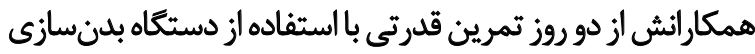

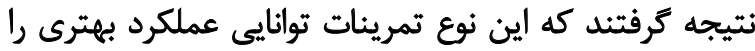

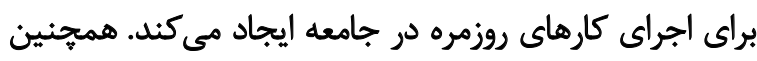

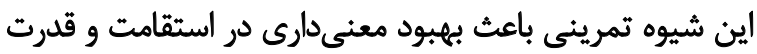

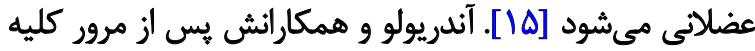

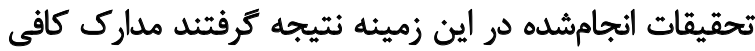

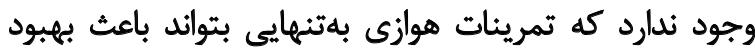

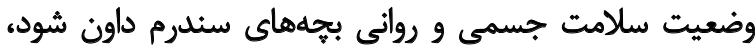

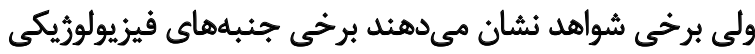

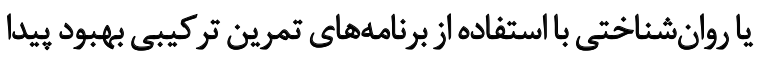

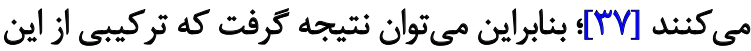

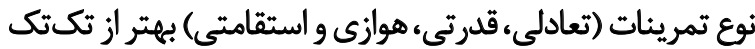

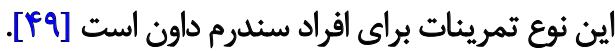

برخى تحقيقات بهبود معنى دار استقامت عضلانى افراد با سندرم

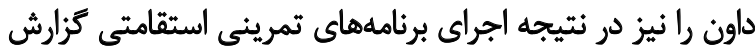

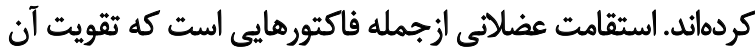

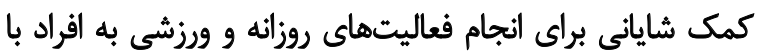

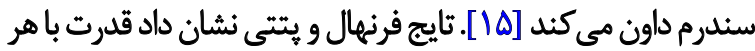

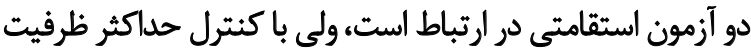

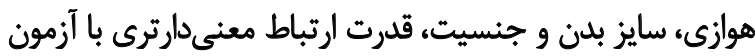

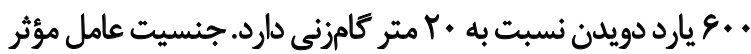

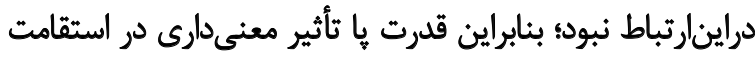
دويدن در افراد كمتوان ذهنى خفيف تا متوسط دارد [• [ه]. تعادل و كثترل ياسجر تعادل از عوامل آمادكى جسمائى مرتبط با سلامت نيست، اما

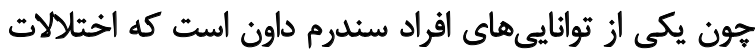

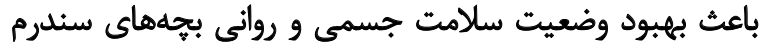

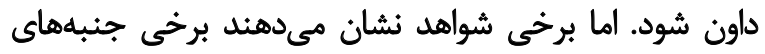

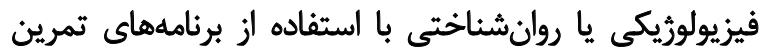

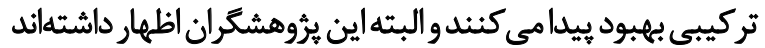

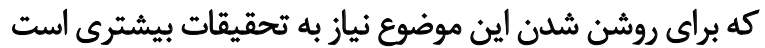

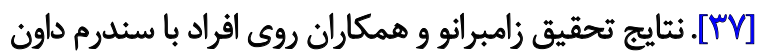

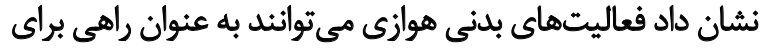

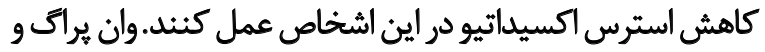

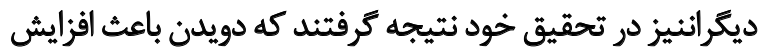

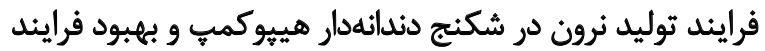

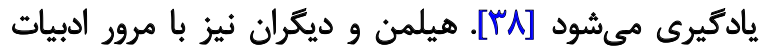

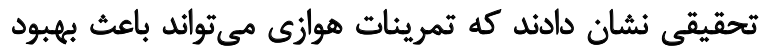

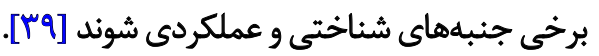

تمرينات قدرتى و تمرينات ثركيبى در افراد با سندرم داون از جمله مهمترين نوع تمرينات مورداستفاده براى افراد با

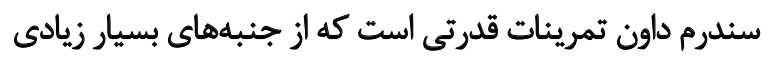

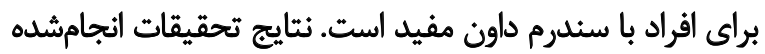

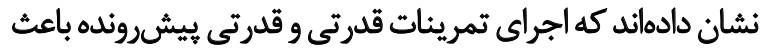

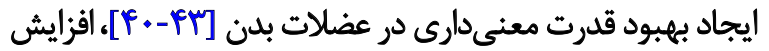

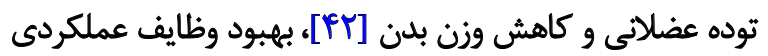

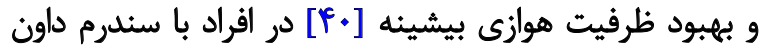

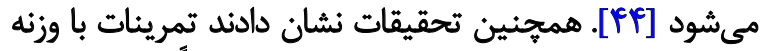

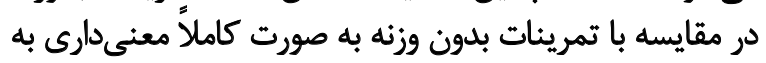

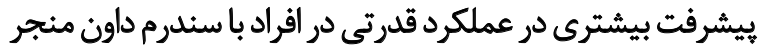

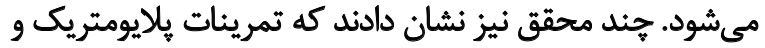

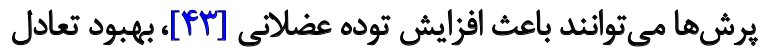

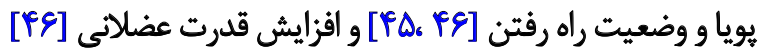

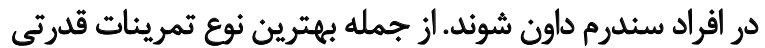

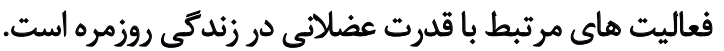

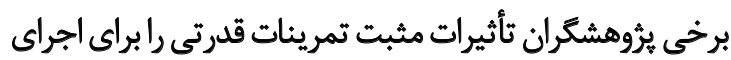

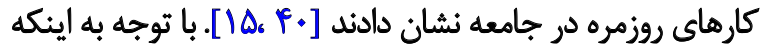

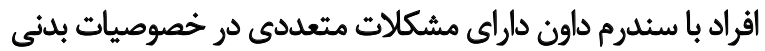

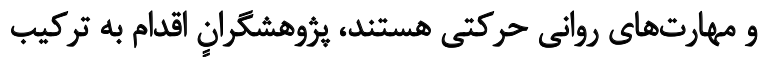

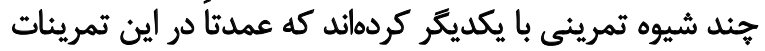

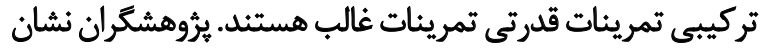

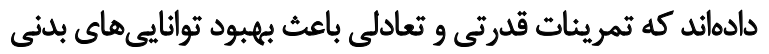

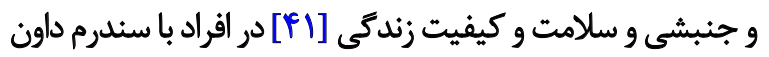

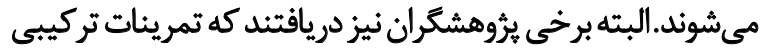

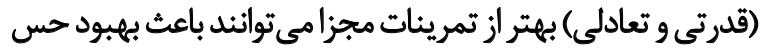

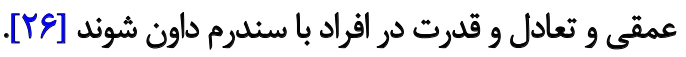
همجئين جنانجه تمرينات قدرتى با تمرينات خابكى همراه

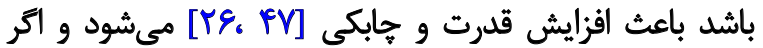


از تجارب محيطى و برنامههاي تمرينى در افراد مبتلا به سندرم

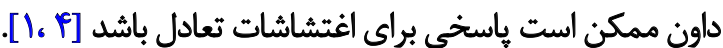

ت تركيب بدنى

در تحقيقات مختلف، كودكان مبتلا به سندرم داون را افرادى

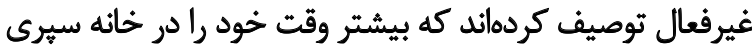

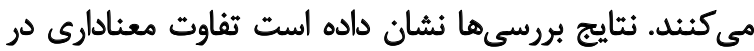

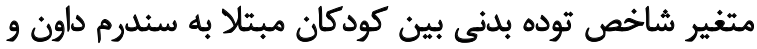

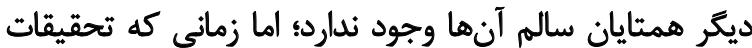

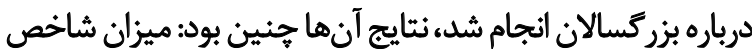

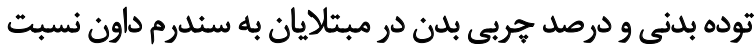

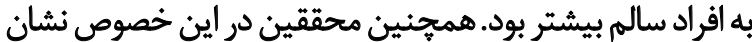

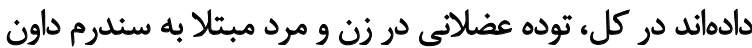

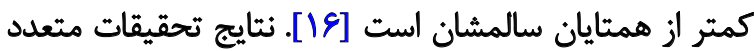

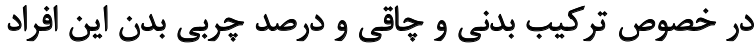

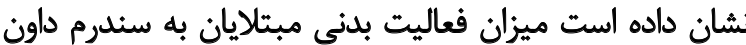

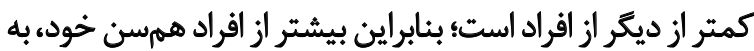

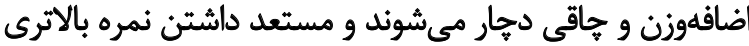

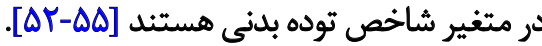
زمان شروع اضافهوزن اين افراد، عمدتاً اوايل كودكى استى

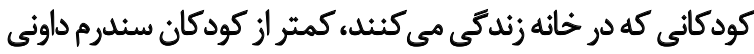

بيشترى دارد، در اين تحقيق نيز مورد ارزيابى قرار كرفت. افراد

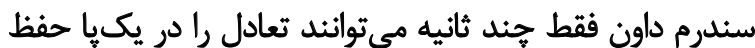

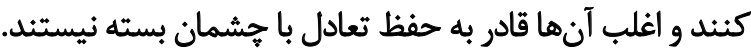

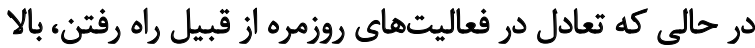

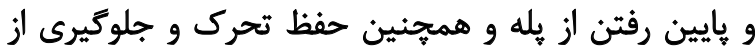

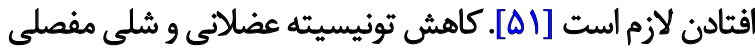

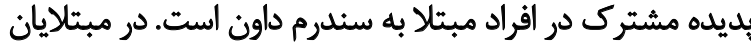

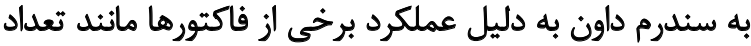

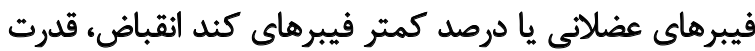

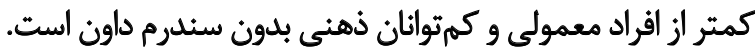

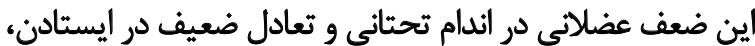

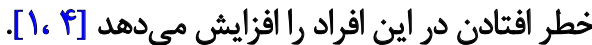
در جندين مطالعه تأثير مثبت برنامههاى تمرينى راه رفتن در

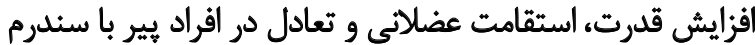

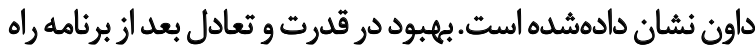

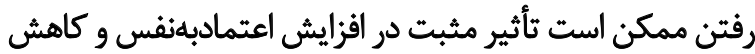

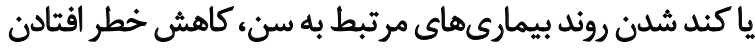

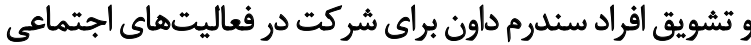

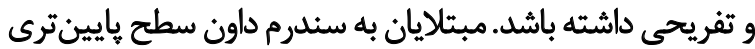

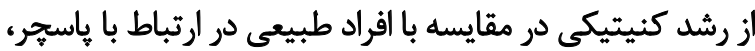

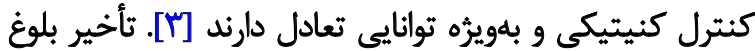

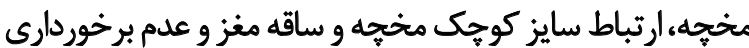

جدول 8. خلاصه نتايج آمادگى جسمانى در افراد سندرم داون

\begin{tabular}{|c|c|}
\hline 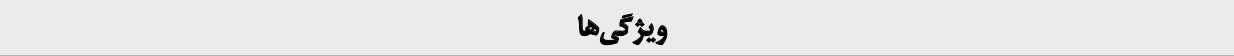 & توضيحات \\
\hline 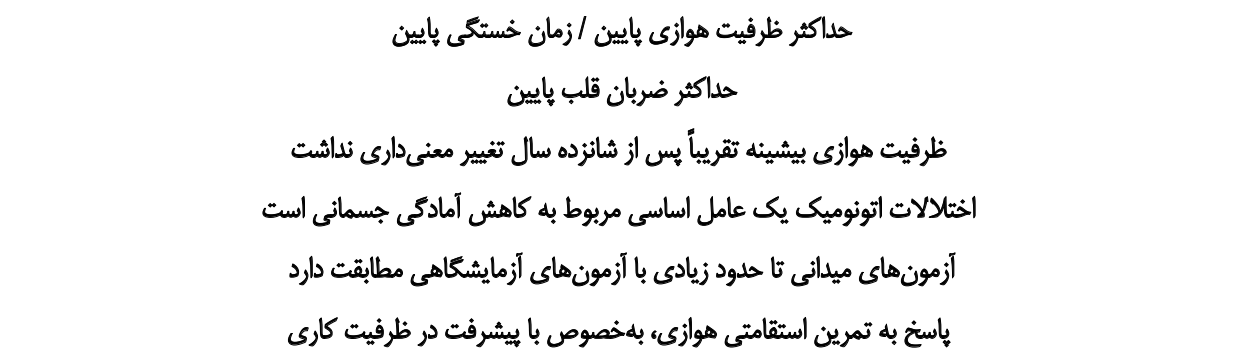 & قلبىعروقى \\
\hline 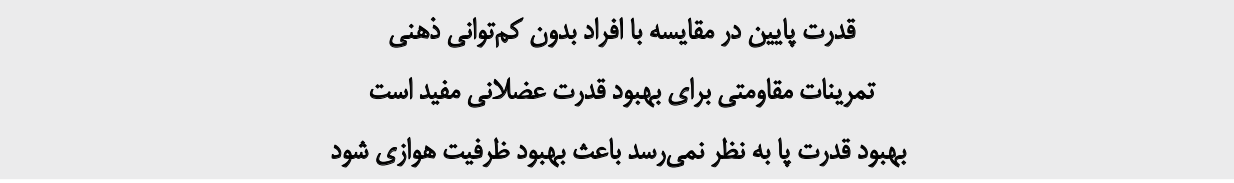 & قدرت عضلانى \\
\hline 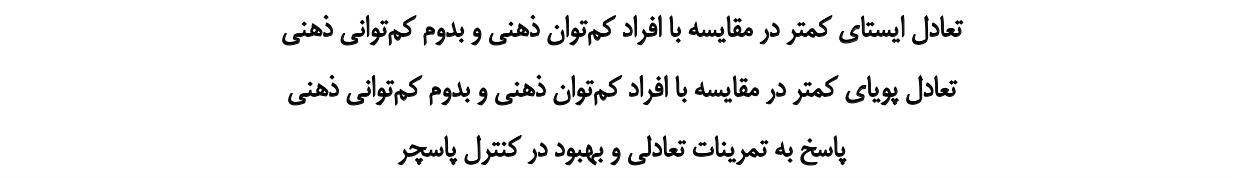 & كثترل ياسجر \\
\hline 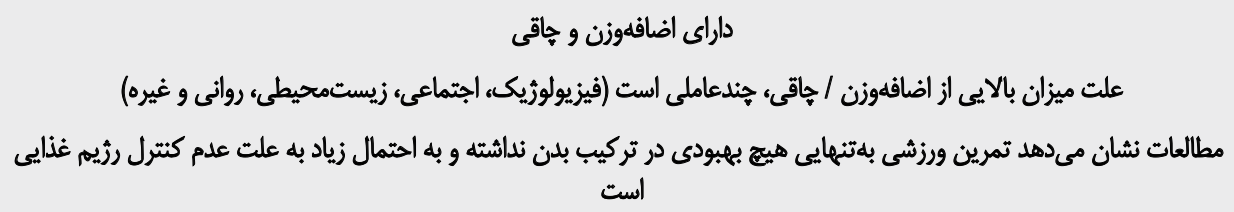 & تركيب بلنئ \\
\hline
\end{tabular}

مجله بيومكانيك وزنث 


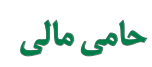

اين تحقيق هيج كمك مالى خاصى از سازمانهاي عمومى يا

$$
\text { خصوصى دريافت نكرده است. }
$$

$$
\text { مشاركت نويسند مَّان }
$$

مفهومسازى، روششناسى و نظارت: تمامى نويسندكان؛

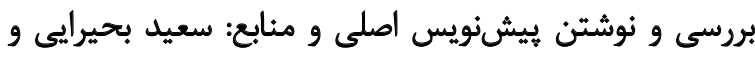
رحمان اميرى؛ مرور و بررسى و ويرايش: حسن دانشمندى. برئ

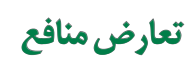

بنابر اظهار نويسندكان اين مقاله تعارض منافع ندارد.

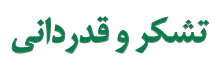

بدين وسيله نويسندكان از مسئولين يُروهشى و آموزشي توري

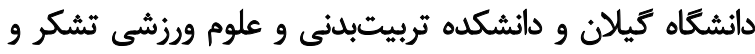
قدردانى مي كنيند.

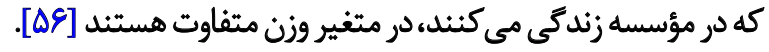

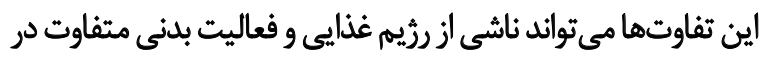

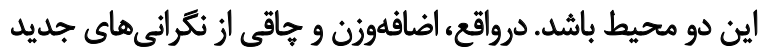

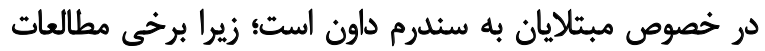

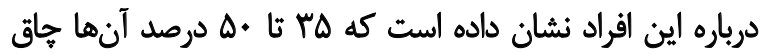

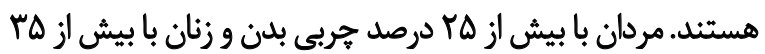

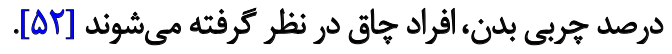

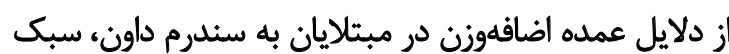

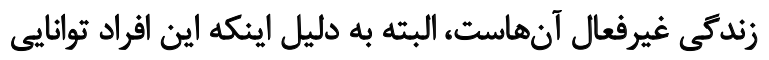

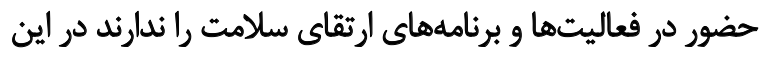

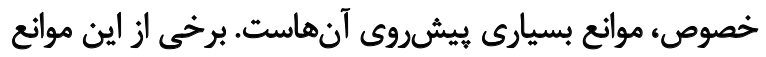

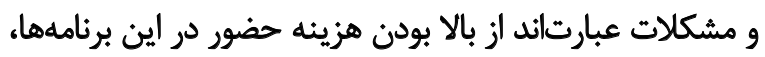

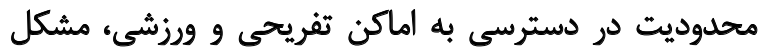

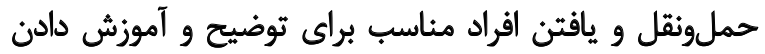

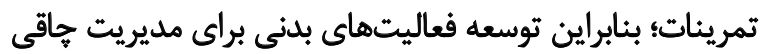

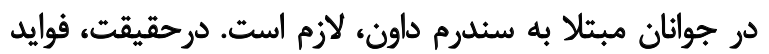

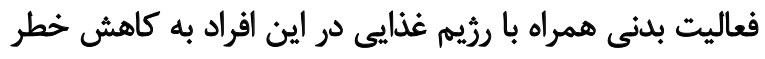

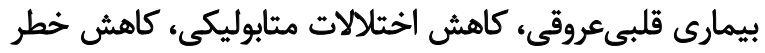

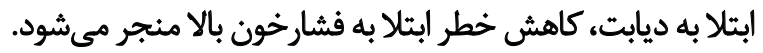

\section{تتيجهَيرى نهايى}

با توجه به نتايج تحقيقات مختلف، كودكان و نوجوانان مبتلا

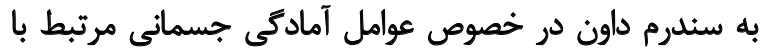

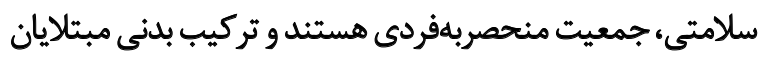

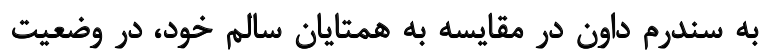

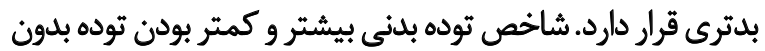

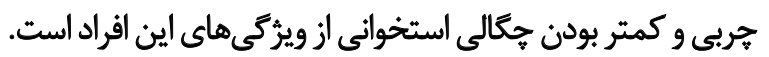

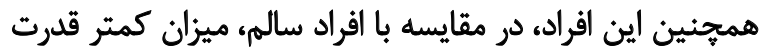

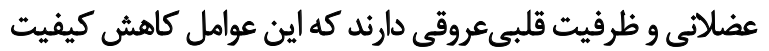

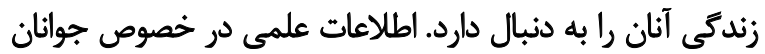

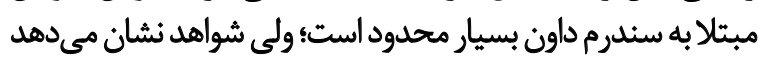

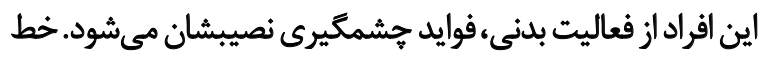

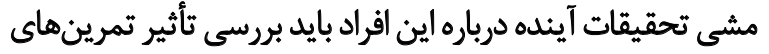

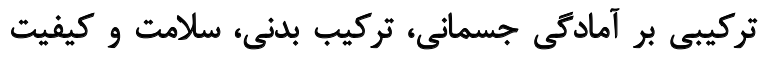

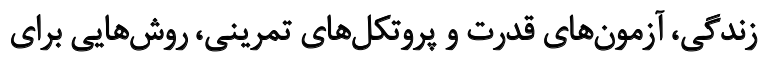

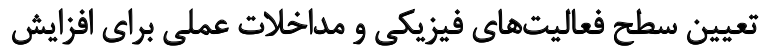

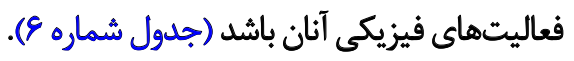

مالاحظات اخلاقي

$$
\begin{aligned}
& \text { ييروى از اصول اخلاق يثوهش } \\
& \text { ايـن مقالـه از نــــ مـرورى اسـت و نمونـهـ انسـانى و حيوانـى } \\
& \text { نداشــــه است. }
\end{aligned}
$$




\section{References}

[1] Bahiraei S, Daneshmandi H. [The Study of relationship between structural profiles and postural control in individual with Down syndrome (Persian)]. JPSBS. 2014; 2(4):21-32. [DOI: 10.22077/JPSBS.2014.19]

[2] Rahmani P, Shahrokhi H, Daneshmandi H. [The investigation of spinal abnormalities \& balance and relation between them in patients with Down Syndrome (Persian)]. MRJ. 2014; 8(4):63-9. http://mrj.tums.ac.ir/ article-1-5175-en.htm

[3] Rahmani P SH. [The study of static and dynamic balance in mentally retarded female students with and without Down Syndrome (DS) (Persian)]. JESM. 2011; 2(2):97-113. https://jsmed.ut.ac.ir/article_24147. html

[4] Bahiraei S, Daneshmandi H, Karimi N. The relationship between alignment of upper limb and postural control in adolescents with Down Syndrome. Med Sport. 2014; 10(2):2322-9.

[5] Daneshmandi H, Bahiraei S, karimi N, babakhani M. [Epidemiology of malalignment Head, Neck, Shoulders and Spine in individuals with Down Syndrome (Persian)]. PTJ. 2013; 2(2):81-9. http://ptj.uswr.ac.ir/ article-1-103-en.html

[6] Farin Soleimani ZM. Down Syndrome: Modern methods in care and treatment(1). 2ed. Tehran: University of Rehabilitation Sciences and Social Welfare; 2011.

[7] Torr J, Strydom A, Patti P, Jokinen N. Aging in Down Syndrome: Morbidity and mortality. J Policy Pract Intellect Disabil. 2010; 7(1):70-81. [DOI:10.1111/j.1741-1130.2010.00249.x]

[8] Sarlak Z, Kashi A, Shariati Junaid M.,. the selected training program on cardiac function in adults with Down Syndrome. Sport Physiology. 2013; 5(19):15-32. https://spj.ssrc.ac.ir/article_55.html

[9] Temple VA, Frey GC, Stanish HI. Physical activity of adults with mental retardation: Review and research needs. Am J Health Promot. 2006; 21(1):2-12. [DOI:10.4278/0890-1171-21.1.2] [PMID]

[10] Looper J, Ulrich DA. Effect of treadmill training and supramalleolar orthosis use on motor skill development in infants with Down syndrome: a randomized clinical trial. Phys Ther. 2010; 90(3):382-90. [DOI:10.2522/ ptj.20090021] [PMID]

[11] Whitt-Glover MC, O'Neill KL, Stettler N. Physical activity patterns in children with and without Down Syndrome. Pediatr Rehabil. 2006; 9(2):158-64. [DOI:10.1080/13638490500353202] [PMID]

[12] Esposito PE, MacDonald M, Hornyak JE, Ulrich DA. Physical activity patterns of youth with Down Syndrome. Intellect Dev Disabil. 2012; 50(2):109-19. [DOI:10.1352/1934-9556-50.2.109] [PMID]

[13] Shields N, Dodd KJ, Abblitt C. Do children with Down syndrome perform sufficient physical activity to maintain good health? A pilot study. Adapt Phys Activ Q. 2009; 26(4):307-20. [DOI:10.1123/apaq.26.4.307] [PMID]

[14] American College of Sports Medicine. ACSM's health-related physical fitness assessment. Philadelphia: Lippincott Williams \& Wilkins; 2013. https://shop.lww.com/ACSM-s-Health-Related-Physical-FitnessAssessment/p/9781496338808

[15] Shields N, Taylor NF, Dodd KJ. Effects of a community-based progressive resistance training program on muscle performance and physical function in adults with Down Syndrome: A randomized controlled trial. Arch Phys Med Rehabil. 2008; 89(7):1215-20. [DOI:10.1016/j. apmr.2007.11.056] [PMID]
[16] González-Agüero A, Vicente-Rodríguez G, Moreno LA, Guerra-Balic M, Ara I, Casajus JA. Health-related physical fitness in children and adoles cents with Down Syndrome and response to training. Scand J Med Sci Sports. 2010; 20(5):716-24. [DOI:10.1111/j.1600-0838.2010.01120.x] [PMID]

[17] Fernhall B, Pitetti KH, Rimmer JH, McCUBBIN JA, Rintala P, Millar AL, et al. Cardiorespiratory capacity of individuals with mental retardation including Down syndrome. Med Sci Sports Exerc. 1996; 28(3):366-71. [DOI:10.1097/00005768-199603000-00012] [PMID]

[18] Varela AM, Bettencount Sardinha L, Pitetti KH. Effects of an aerobic rowing training regimen in young adults with Down Syndrome. Am J Ment Retard. 2001; 106(2):135-44. [DOI:10.1352/08958017(2001)1062.0.CO;2]

[19] González-Agüero A, Gómez-Cabello A, Matute-Llorente A, GómezBruton A, Vicente-Rodríguez G, Casajús JA. Effects of a circuit training including plyometric jumps on cardiorespiratory fitness of children and adolescents with Down Syndrome. Rev Med Int Sindr Down. 2014; 18(3):35-42. [DOI:10.1016/S2171-9748(14)70053-4]

[20] Matute-Llorente A, González-Agüero A, Gómez-Cabello A, VicenteRodríguez G, Casajús JA. Physical activity and cardiorespiratory fitness in adolescents with Down Syndrome. Nutr Hosp. 2013; 28(4):1151-5. [DOI: 10.3305/nh.2013.28.4.6509] [PMID]

[21] Wee SO, Pitetti KH, Goulopoulou S, Collier SR, Guerra M, Baynard T. Impact of obesity and Down Syndrome on peak heart rate and aerobic capacity in youth and adults. Res Dev Disabil. 2015; 36:198-206. [DOI:10.1016/j.ridd.2014.10.002] [PMID]

[22] Lewis CL, Fragala-Pinkham MA. Effects of aerobic conditioning and strength training on a child with Down Syndrome: A case study. Pediatr Phys Ther. 2005; 17(1):30-6. [DOI:10.1097/01.PEP.0000154185.55735. A0] [PMID]

[23] Shields N, Taylor NF. A student-led progressive resistance training program increases lower limb muscle strength in adolescents with Down Syndrome: A randomised controlled trial. J Physiother. 2010; 56(3):18793. [DOI:10.1016/S1836-9553(10)70024-2] [PMID]

[24] Cowley PM, Ploutz-Snyder LL, Baynard T, Heffernan KS, Young Jae S, Hsu S, et al. The effect of progressive resistance training on leg strength, aerobic capacity and functional tasks of daily living in persons with Down Syndrome. Disabil Rehabil. 2011; 33(23-24):2229-36. [DOI:10.310 9/09638288.2011.563820] [PMID]

[25] Gupta S, krishna Rao B, Kumaran SD. Effect of strength and balance training in children with Down's Syndrome: A randomized controlled trial. Clin Rehabil. 2011; 25(5):425-32. [DOI:10.1177/0269215510382929] [PMID]

[26] Lin H-C, Wuang Y-P. Strength and agility training in adolescents with Down Syndrome: A randomized controlled trial. Res Dev Disabil. 2012; 33(6):2236-44. [DOI:10.1016/j.ridd.2012.06.017] [PMID]

[27] Chen H-L, Yeh C-F, Howe T-H. Postural control during standing reach in children with Down Syndrome. Res Dev Disabil. 2015; 38:345-51. [DOI:10.1016/j.ridd.2014.12.024] [PMID]

[28] Galli M, Rigoldi C, Mainardi L, Tenore N, Onorati P, Albertini G. Postural control in patients with Down Syndrome. Disabil Rehabil. 2008; 30(17):1274-8. [DOI:10.1080/09638280701610353] [PMID]

[29] Rigoldi C, Galli M, Mainardi L, Crivellini M, Albertini G. Postural contro in children, teenagers and adults with Down syndrome. Res Dev Disabil. 2011;32(1):170-5. [DOI:10.1016/j.ridd.2010.09.007] [PMID]

[30] Meneghetti CHZ, Blascovi-Assis SM, Deloroso FT, Rodrigues GM. Static balance assessment among children and adolescents with Down 
Syndrome. Rev Bras Fisioter. 2009; 13(3):230-5. [DOI:10.1590/S141335552009005000029]

[31] Frey GC, Chow B. Relationship between BMI, physical fitness, and motor skills in youth with mild intellectual disabilities. Int J Obes (Lond). 2006; 30(5):861-7. [DOI:10.1038/sj.ijo.0803196] [PMID]

[32] Rubin SS, Rimmer JH, Chicoine B, Braddock D, McGuire DE. Overweight prevalence in persons with Down Syndrome. Ment Retard. 1998; 36(3):175-81. [DOI:10.1352/0047-6765(1998)0362.0.CO;2] [PMID]

[33] Jimenez L, Cerda J, Alberti G, Lizama M. High rates of overweight and obesity in Chilean children with Down Syndrome. Rev Med Chil. 2015; 143(4):451-8. [DOI:10.4067/\$0034-98872015000400006][PMID]

[34] van Gameren-Oosterom HBM, van Dommelen $P$, Schönbeck $Y_{\text {f }}$ Oudesluys-Murphy AM, van Wouwe JP, Buitendijk SE. Prevalence of overweight in Dutch children with Down Syndrome. Pediatrics. 2012; 130(6):e1520-6. [DOI:10.1542/peds.2012-0886] [PMID]

[35] Pastore E, Marino B, Calzolari A, Digilio MC, Giannotti A, Turchetta A. Clinical and cardiorespiratory assessment in children with Down Syndrome without congenital heart disease. Arch Pediatr Adolesc Med. 2000; 154(4):408-10. [DOI:10.1001/archpedi.154.4.408] [PMID]

[36] Giagoudaki F, Dimitros E, Kouidi E, Deligiannis A. Effects of exercise training on heart-rate-variability indices in individuals with Down Syndrome. J Sport Rehabil. 2010; 19(2):173-83. [DOI:10.1123/jsr.19.2.173] [PMID]

[37] Andriolo RB, El Dib RP, Ramos L, Atallah ÁN, da Silva EM. Aerobic exercise training programmes for improving physical and psychosocial health in adults with Down Syndrome. Cochrane Database Syst Rev. 2010; (5):CD005176. [DOI:10.1002/14651858.CD005176.pub4] [PMID]

[38] Van Praag H, Christie BR, Sejnowski TJ, Gage FH. Running enhances neurogenesis, learning, and long-term potentiation in mice. Proc Natl Acad Sci USA. 1999; 96(23):13427-31. [DOI:10.1073/pnas.96.23.13427] [PMID] [PMCID]

[39] Hillman CH, Erickson KI, Kramer AF. Be smart, exercise your heart: exercise effects on brain and cognition. Nat Rev Neurosci. 2008; 9(1):5865. [DOI:10.1038/nrn2298] [PMID]

[40] Cowley PM, Ploutz-Snyder LL, Baynard T, Heffernan K, Jae SY, Hsu S, et al. Physical fitness predicts functional tasks in individuals with Down Syndrome. Med Sci Sports Exerc. 2010; 42(2):388-93. [DOI:10.1249/ MSS.0b013e3181b07e7a] [PMID]

[41] Tsimaras VK, Fotiadou EG. Effect of training on the muscle strength and dynamic balance ability of adults with Down Syndrome. Strength Cond Res. 2004; 18(2):343-7. [DOI:10.1519/00124278200405000-00025] [PMID]

[42] Rimmer JH, Heller T, Wang E, Valerio I. Improvements in physical fitness in adults with Down Syndrome. Am J Ment Retard. 2004; 109(2):165-74. [DOI:10.1352/0895-8017(2004)1092.0.CO;2] [PMID]

[43] Shields N, Taylor NF, Wee E, Wollersheim D, O'Shea SD, Fernhall B. A community-based strength training programme increases muscle strength and physical activity in young people with Down Syndrome: a randomised controlled trial. Res Dev Disabil. 2013; 34(12):4385-94. [DOI:10.1016/j.ridd.2013.09.022] [PMID]

[44] Weber R, French R. Down's Syndrome adolescents and strength training. Clin Kinesiol. 1998; 42:13-21.

[45] Wang WY, Chang JJ. Effects of jumping skill training on walking balance for children with mental retardation and Down's Syndrome. Kaohsiung J Med Sci. 1997; 13(8):487-95. [PMID]
[46] Wang W-Y, Ju Y-H. Promoting balance and jumping skills in children with Down Syndrome. Percept Mot Skills. 2002; 94(2):443-8. [DOI:10.2466/pms.2002.94.2.443] [PMID]

[47] Mendonca GV, Pereira FD, Fernhall B. Effects of combined aerobic and resistance exercise training in adults with and without Down Syndrome. Arch Phys Med Rehabil. 2011; 92(1):37-45. [DOI:10.1016/j. apmr.2010.09.015] [PMID]

[48] Shields N, Taylor NF, Fernhall B. A study protocol of a randomised controlled trial to investigate if a community based strength training programme improves work task performance in young adults with Down Syndrome. BMC pediatr. 2010; 10(1):1. [DOI:10.1186/1471-2431-10-17] [PMID] [PMCID]

[49] Leavey VJ, Sandrey MA, Dahmer G. Comparative effects of 6-week balance, gluteus medius strength, and combined programs on dynamic postural control. J Sport Rehabil. 2010; 19(3):268-87. [DOI:10.1123/ jsr.19.3.268] [PMID]

[50] Fernhall B, Pitetti KH. Leg strength is related to endurance run performance in children and adolescents with mental retardation. Pediatr Exerc Sci. 2000; 12(3):324-33. [DOI:10.1123/pes.12.3.324]

[51] Oja P, Tuxworth B. Eurofit for adults: A test battery for the assessment of the health-related fitness of adults. Strassbourg: Council of Europe, Committee for the Development of Sport; 1995. p. 134-89

[52] Barnhart RC, Connolly B. Aging and Down Syndrome: Implications for physical therapy. Phys Ther. 2007; 87(10):1399-406. [DOI:10.2522/ ptj.20060334] [PMID]

[53] Harris N, Rosenberg A, Jangda S, O'Brien K, Gallagher ML. Prevalence of obesity in International Special Olympic athletes as determined by body mass index. J Am Diet Assoc. 2003; 103(2):235-7. [DOI:10.1053/ jada.2003.50025] [PMID]

[54] Murray J, Ryan-Krause P. Obesity in children with Down Syndrome: background and recommendations for management. Pediatr Nurs. 2010; 36(6):314-9. [PMID]

[55] Melville CA, Cooper S-A, McGrother CW, Thorp CF, Collacott R. Obesity in adults with Down Syndrome: A case-control study. J Intellect Disabil Res. 2005; 49(Pt 2):125-133. [DOI:10.1111/j.1365-2788.2004.00616.x] [PMID]

[56] Boer P. The functional fitness capacity of adults with Down Syndrome in South Africa. [Doctoral dissertation]. Stellenbosch: University of Stellenbosch; 2010. 